\title{
The maximum principle and the Dirichlet problem for Dirac-harmonic maps
}

\author{
Qun Chen · Jürgen Jost • Guofang Wang
}

Received: 10 May 2011 / Accepted: 23 January 2012 / Published online: 29 March 2012

(C) The Author(s) 2012. This article is published with open access at Springerlink.com

\begin{abstract}
We establish a maximum principle and uniqueness for Dirac-harmonic maps from a Riemannian spin manifold with boundary into a regular ball in any Riemannian manifold $N$. Then we prove an existence theorem for a boundary value problem for Dirac-harmonic maps.
\end{abstract}

Keywords Dirac-harmonic map $\cdot$ Maximum principle $\cdot$ Uniqueness $\cdot$ Existence

Mathematics Subject Classification Primary 58E20 - Secondary 53C27

\section{Introduction}

Dirac-harmonic maps have been introduced in $[8,9]$ as mathematical versions (without anticommuting variables) of the supersymmetric $\sigma$-model of high energy theoretical physics. This $\sigma$-model is an important model in quantum field theory that allows for a systematic study

Communicated by L. Ambrosio.

\section{Q. Chen}

School of Mathematics and Statistics, Wuhan University, Wuhan, 430072, People's Republic of China e-mail: qunchen@whu.edu.cn

Q. Chen · J. Jost ( $ه)$

Max Planck Institute for Mathematics in the Sciences, Inselstr. 22, 04103 Leipzig, Germany e-mail: jost@mis.mpg.de

J. Jost

Department of Mathematics and Computer Science, University of Leipzig, 04081 Leipzig, Germany

G. Wang

Institute of Mathematics, University Freiburg, Eckerstr. 1, 79104 Freibrug, Germany

e-mail: guofang.wang@math.uni-freiburg.de 
of many phenomena that also occur in other, more difficult models, like supersymmetric Yang-Mills. We refer to [22] for a systematic presentation of this model. In mathematical terms, the model couples a harmonic map type nonlinear field and a spinor field along that map that solves a nonlinear Dirac equation. In the same manner that the theory of ordinary harmonic maps both found important geometric applications and paved the way for the mathematical analysis of other geometric variational problems like Yang-Mills, we hope that Dirac-harmonic maps can play a similarly useful role. In order to start such a program in more concrete terms, we therefore need to carefully develop the appropriate analytical tools. Now the most challenging analytical problem for ordinary harmonic maps has been the existence problem for such maps with values in manifolds that may have positive sectional curvature, and here, the most powerful analytical tools in the theory have been developed, see $[15,16,19,20,23,24,29]$ and others. The result achieved here is that the Dirichlet problem for harmonic maps can be (uniquely) solved when the target is confined to a strictly convex ball, and this result is optimal, as observed in [16].

We attempt a similar analysis here. Our program is more difficult than the classical theory for several reasons. For instance, as we shall explain below, we can neither employ variational methods nor heat equation techniques. We therefore need to combine some of the achievements and techniques of the classical theory, in particular the maximum principle of Jäger-Kaul $[18,19]$ with new estimates for nonlinear Dirac equations and handle the coupling between the fields in a rather subtle manner. The maximum principle will then allow us to derive a-priori estimates that we can utilize for a continuity method to obtain an existence result for a boundary value problem that combines Dirichlet data for the harmonic map type field with a boundary condition for the spinor field. The continuity scheme is inspired by that of von Wahl [31], but we still need to handle a number of geometric aspects that played no role for the analytical purposes of von Wahl.

\subsection{Dirac-harmonic maps}

In this subsection, we first recall the definition of Dirac-harmonic maps. Let $\left(M^{n}, g\right)$ be a Riemannian manifold with a fixed spin structure, and let $\Sigma M$ be its spinor bundle, on which we choose a Hermitian metric $\langle\cdot, \cdot\rangle$. The Levi-Civita connection $\nabla$ of $g$ induces an connection (which we still denote by $\nabla$ ) on $\Sigma M$ compatible with $\langle\cdot, \cdot\rangle$ and $g$. Let $\phi$ be a smooth map from $M$ to a Riemannian manifold $(N, h)$ of dimension $n^{\prime} \geq 2$ and $\phi^{-1} T N$ the pull-back bundle of $T N$ by $\phi$. On the twisted bundle $\Sigma M \otimes \phi^{-1} T N$ there is a Hermitian metric (still denoted by $\langle\cdot, \cdot\rangle)$ induced from the metrics on $\Sigma M$ and $\phi^{-1} T N$ and a natural connection $\widetilde{\nabla}$ on $\Sigma M \otimes \phi^{-1} T N$ induced from those on $\Sigma M$ and $\phi^{-1} T N$.

In local coordinates $\left\{x_{\alpha}\right\}$ and $\left\{y^{i}\right\}$ on $M$ and $N$ respectively, one can write the section $\psi$ of $\Sigma M \otimes \phi^{-1} T N$ as

$$
\psi(x)=\psi^{j}(x) \otimes \partial_{y^{j}}(\phi(x)),
$$

where $\psi^{i}$ is a (local) spinor field on $M$ and $\left\{\partial_{y^{j}}\right\}$ is a local basis on $N$. The connection $\widetilde{\nabla}$ can be written as

$$
\widetilde{\nabla} \psi(x)=\nabla \psi^{i}(x) \otimes \partial_{y^{i}}(\phi(x))+\Gamma_{j k}^{i} \nabla \phi^{j}(x) \psi^{k}(x) \otimes \partial_{y^{i}}(\phi(x)) .
$$

Here and in the sequel, we use the summation convention. $\psi$ is a spinor field along the map $\phi$. The Dirac operator along the map $\phi$ is defined [8] as

$$
\begin{aligned}
\not \partial \psi & :=e_{\alpha} \cdot \widetilde{\nabla}_{e_{\alpha}} \psi \\
& =\not \partial \psi^{i}(x) \otimes \partial_{y^{i}}(\phi(x))+\Gamma_{j k}^{i} \nabla_{e_{\alpha}} \phi^{j}(x) e_{\alpha} \cdot \psi^{k}(x) \otimes \partial_{y^{i}}(\phi(x)),
\end{aligned}
$$


where $\left\{e_{\alpha}\right\}$ is the local orthonormal basis of $M$ and

$$
\not \partial:=e_{\alpha} \cdot \nabla_{e_{\alpha}}
$$

is the usual Dirac operator on $M$. We use $X \cdot \Psi$ or $\gamma(X) \Psi$ to denote the Clifford multiplication of a smooth vector field $X \in \Gamma(M)$ and spinor field $\Psi \in \Gamma(\Sigma M)$ on $M$.

Motivated by the supersymmetric version of the $\sigma$-model (harmonic maps), we introduced in [8] the following functional

$$
L(\phi, \psi):=\frac{1}{2} \int_{M}\left(|d \phi|^{2}+\langle\psi, \not p\rangle\right),
$$

where $\langle\psi, \xi\rangle:=h_{i j}(\phi)\left\langle\psi^{i}, \xi^{j}\right\rangle$, for $\psi, \xi \in \Gamma\left(\Sigma M \otimes \phi^{-1} T N\right)$. Since the value of $\int_{M}\langle\psi, D$ $\psi\rangle$ is real, in the sequel we replace the Hermitian product $\langle\cdot, \cdot\rangle$ by its real part (but still use the same notation). The Euler-Lagrange system for $L$ is (see [8])

$$
\begin{aligned}
\tau^{i}(\phi) & =\frac{1}{2} R_{j k l}^{i}(\phi)\left\langle\psi^{k}, \nabla \phi^{j} \cdot \psi^{l}\right\rangle, \\
\not \psi^{i} & :=\not \partial \psi^{i}+\Gamma_{j k}^{i}(\phi) \partial_{\alpha} \phi^{j} e_{\alpha} \cdot \psi^{k}=0,
\end{aligned}
$$

$i=1,2, \cdots, n^{\prime}:=\operatorname{dim} N$, where $\tau(\phi)$ is the tension field of the map $\phi$.

Set

$$
\mathcal{R}(\phi, \psi):=\frac{1}{2} R^{i}{ }_{j k l}(\phi)\left\langle\psi^{k}, \nabla \phi^{j} \cdot \psi^{l}\right\rangle \otimes \partial_{y^{i}} .
$$

Then (1.2) and (1.3) can be written in a concise form

$$
\begin{aligned}
\tau(\phi) & =\mathcal{R}(\phi, \psi), \\
D \psi & =0 .
\end{aligned}
$$

Solutions $(\phi, \psi)$ of (1.4), (1.5) are called Dirac-harmonic maps (c.f. [8], [9]). Dirac-harmonic maps include two types of solutions that are trivial from our present point of view. One is $(\phi, 0)$ with a harmonic map $\phi$ and another one is $(p, \psi)$, where $p$ stands for a constant map to a some point $p \in N$ and $\psi$ is a tuple of ordinary harmonic spinor fields. We are interested in solutions that couple the fields $\phi$ and $\psi$. Examples of such non-trivial Dirac-harmonic maps were constructed in [8] by using twistor spinors and conformal maps between spheres. This construction was generalized in [25] to obtain more non-trivial solutions.

The study of Dirac-harmonic maps has been taken up by several mathematicians. The regularity theory has been developed in [8], [9], [32], [35] and [10]. The blow-up analysis was established in [8], [9] and [34]. See also [33] for a classification result for Dirac-harmonic maps. However, one of the most important problems, the general existence problem, still remains open. Compared with the case of harmonic maps, the existence problem here is technically much more difficult. First, since the functional $L$ is unbounded both from above and from below, one cannot use the direct methods of the calculus of variations to obtain solutions, though we already possess the regularity theory. For the same reason, the wellknown existence scheme of Sacks and Uhlenbeck in [30], which is based on considering the minimizers of perturbed functionals, seems also not applicable here. The heat flow method, which is a powerful tool for many problems in geometric analysis, does not work either, since the equation for the spinor field is of first order. (Perhaps one can consider to deform the equation for the spinor field by using a $1 / 2$ order nonlocal operator.).

The method of continuity and the Leray-Schauder theory are other fundamental tools for dealing with existence problems. To apply these theories, one needs first to establish suitable 
a priori estimates for solutions, so as to guarantee some compactness. This is one of the main objectives of this paper. In general, as in the theory of harmonic maps one cannot expect that a priori estimates hold for Dirac-harmonic maps without any constraint. In this paper, we derive a priori estimates for Dirac-harmonic maps with image in a small ball, by generalizing the maximum principle of Jäger-Kaul [19] for harmonic maps. With these a priori estimates we can then show the existence and uniqueness of the Dirichlet problem for Dirac-harmonic maps with small boundary data. The precise results are stated in the next subsection.

\subsection{Main results}

We consider the boundary value problem for Dirac-harmonic maps. We first establish a maximum principle and then prove an existence theorem. Let $M$ be a compact Riemannian manifold with boundary $\partial M, B_{R}\left(y_{0}\right)$ a geodesic ball centered at $y_{0}$ in an arbitrary Riemannian manifold $N$ whose sectional curvature is bounded from above by a constant $\kappa>0$, where the radius of $B_{R}\left(y_{0}\right)$ satisfies $R<2 \pi / \sqrt{\kappa}$. We call $B_{R}\left(y_{0}\right)$ a regular ball, and assume that it satisfies the cut-locus condition (c.f. [19]), that is, any pair of points in the set can be joined by a unique minimal geodesic arc.

We will consider Dirac-harmonic maps $(\phi, \psi)$ from $M$ to $N$ satisfying

$$
\phi(M) \subset B_{R}\left(y_{0}\right), \quad|\psi|^{2}<C_{0}
$$

for some positive constants $R$ and $C_{0}$.

For our purpose, we may assume that

$$
R<\left(1-\varepsilon_{0}\right) \cdot \frac{\pi}{2 \sqrt{\kappa}}, \quad C_{0} \leq 1
$$

for some constant $\varepsilon_{0} \in(0,1)$.

By the assumption of the regular ball, on $B_{R}\left(y_{0}\right)$ we can choose normal coordinates $\left\{y^{i}\right\}_{i=1,2, \cdots, n^{\prime}}$ centered at $y_{0}$. In this coordinate system,

$$
\phi:=\left(\phi^{1}, \cdots, \phi^{n^{\prime}}\right), \quad \psi=\psi^{i}(x) \otimes \frac{\partial}{\partial y^{i}}(\phi(x)) .
$$

For simplicity, we write

$$
\phi:=\left(\phi^{1}, \cdots, \phi^{n^{\prime}}\right), \quad \psi:=\left(\psi^{1}, \cdots, \psi^{n^{\prime}}\right) .
$$

We call the above expressions the representation of $(\phi, \psi)$ in the normal coordinate system. Throughout this paper, we will use this representation. Moreover we denote $\left|\phi_{1}-\phi_{2}\right|^{2}:=$ $\sum_{i}\left(\phi_{1}^{i}-\phi_{2}^{i}\right)^{2},\left|\psi_{1}-\psi_{2}\right|^{2}:=\sum_{i}\left|\psi_{1}^{i}-\psi_{2}^{i}\right|^{2}$ and $\left|d \phi_{1}-d \phi_{2}\right|^{2}:=\sum_{i, \alpha}\left(\phi_{1 \alpha}^{i}-\phi_{2 \alpha}^{i}\right)^{2}$.

In [18] and [19], Jäger and Kaul established maximum principles for harmonic maps and solutions of the following nonlinear elliptic systems

$$
\tau(\phi)=b(d \phi),
$$

where $b$ is a given tensor field with quadratic growth in the differential $d \phi$, and satisfies the following Lipschitz condition:

$$
\delta\left(b\left(p_{1}\right), b\left(p_{2}\right)\right) \leq \frac{\lambda}{2} \operatorname{dist}\left(y_{1}, y_{2}\right) \sum_{i=1}^{2}\left|p_{i}\right|^{2}+\mu \delta\left(p_{1}, p_{2}\right)\left(\sum_{i=1}^{2}\left|p_{i}\right|^{2}\right)^{1 / 2}
$$


for some constants $\lambda, \mu \geq 0$ and any pair $p_{1} \in P_{x, y_{1}}, p_{2} \in P_{x, y_{2}}, x \in M$, and $y_{1}, y_{2} \in$ $B_{R}\left(y_{0}\right)$, where $P_{x, y}$ denotes the space of all linear maps of $T_{x} M$ into $T_{y} N$, and $\delta$ denotes the pseudo distance defined by (2.1) and (2.2).

For two solutions $\phi_{1}$ and $\phi_{2}$ of the non-linear elliptic system (1.8) (or two harmonic maps $\left.\phi_{1}, \phi_{2}\right)$, Jäger and Kaul constructed elliptic operators $\mathfrak{L}$ and specific functions $\Theta$ concerning the distance between $\phi_{1}$ and $\phi_{2}$ with $\mathfrak{L}(\Theta) \geq 0$, from which the corresponding maximum principles follow (c.f. [18], [19]). In our case, the Euler-Lagrange equations (1.2), (1.3) yield a system coupling a second order quasi-linear elliptic system and a Dirac type equation on $M$, and this makes the constructions more subtle.

We can try to deal with the $\phi$-part of Dirac-harmonic maps $(\phi, \psi)$ using the methods in [18] and [19]; however, the right hand side of the $\phi$-equation (1.2) does not satisfy (1.9). Nonetheless, we shall be able construct the corresponding elliptic operator $\mathfrak{L}$ and a corresponding function $\Theta$ for Dirac-harmonic maps with the property $\mathfrak{L}(\Theta) \geq 0$, and hence obtain a maximum principle for Dirac-harmonic maps.

For an arbitrary constant $\omega>0$, we let $s_{\omega}$ be the solution of $s_{\omega}^{\prime \prime}+\omega s_{\omega}=0$, that is,

$$
s_{\omega}(t)= \begin{cases}\frac{\sin \sqrt{\omega} t}{\sqrt{\omega}} & \text { if } \quad \omega>0 \\ t & \text { if } \quad \omega=0\end{cases}
$$

Denote

$$
q_{\omega}(t):=\int_{0}^{t} s_{\omega}=\left\{\begin{array}{lll}
\frac{1-\cos \sqrt{\omega} t}{t^{2}} & \text { if } & \omega>0 \\
\frac{t}{2}^{2} & \text { if } & \omega=0 .
\end{array}\right.
$$

Choose $\widetilde{R}:=\frac{1}{1-\varepsilon_{0}} R \in\left(R, \frac{\pi}{2 \sqrt{\kappa}}\right)$. Let $\left(\phi_{1}, \psi_{1}\right)$ and $\left(\phi_{2}, \psi_{2}\right)$ be Dirac-harmonic maps from $M$ to $N$ satisfying (1.6). Define

$$
\Theta:=\frac{q_{\frac{\kappa}{4}}(\rho)+\frac{1}{2}\left|\psi_{1}-\psi_{2}\right|^{2}}{\left\{\left[q_{\kappa}(\widetilde{R})-q_{\kappa}\left(\rho_{1}\right)\right]\left[q_{\kappa}(\widetilde{R})-q_{\kappa}\left(\rho_{2}\right)\right]\left(1+C_{0}-\left|\psi_{1}\right|^{2}\right)\left(1+C_{0}-\left|\psi_{2}\right|^{2}\right)\right\}^{1 / 4}},
$$

where $\rho(x):=\operatorname{dist}\left(\phi_{1}(x), \phi_{2}(x)\right), \rho_{a}(x):=\operatorname{dist}\left(\phi_{a}(x), y_{0}\right), a=1,2, \forall x \in M$. The basic idea here is to compose a strictly convex function (coming from the distance function of the target - that's why we need to assume that the range lies in a convex ball) with the distance between the two maps and likewise to take the squared distance with the spinor fields as some convex operation. Since convex composed with harmonic yields a subharmonic function, we can hope for a maximum principle. Now, there are some substantial technical difficulties with this idea. First of all, the distance between two maps lives on the product of the target with itself, and we need to understand how convexity can be carried over to this product. This issue has been addressed by Jäger-Kaul [18] (and this was substantially harder than what one might have naively expected), the upshot being that we need the counterterms in the denominator. The second difficulty arises from the fact that the map is coupled with a spinor field and therefore no longer harmonic. We thus need to control some error terms. By themselves, as it turns out, they cannot be compensated, however. We rather need to utilize the properties of the Dirac equation for the spinor field in a very careful manner. In fact, due to the nonlinearity of our Dirac equation, we have a third difficulty in estimating norms of the spinor field and controlling additional terms in a Lichnerowicz identity. Fortunately, it turns out that the difficult terms arising from the second and third difficulties can be balanced when we arrange things very carefully. This is very subtle and nontrivial and constitutes perhaps 
the main technical point of this paper. It turns out that we need to assume the spinor field to be sufficiently small in norm; perhaps this can be considered as analogous to the smallness condition on the map, that it be contained in a suitable convex ball in the target.

For the map, we shall assume a Dirichlet type boundary condition. The boundary condition B that we impose on the spinor field will be explained below [see (2.31)].

\section{Theorem 1.1 (Maximum principle and uniqueness for Dirac-harmonic maps) .}

Let $(M, g)$ be a compact Riemannian spin manifold with non-void boundary $\partial M$ which has non-negative mean curvature (w.r.t. the inner normal vector). Assume that $\inf _{M} S_{M}>0$, where $S_{M}$ denotes the scalar curvature of $M$. Let $(N, h)$ be a Riemannian manifold with sectional curvature bounded from above by a constant $\kappa>0$, and $y_{0}$ be any given point in $N$. Then there exist positive constants $R<\pi / 2 \sqrt{\kappa}$ and $C_{0}$ such that for any pair of Diracharmonic maps $\left(\phi_{a}, \psi_{a}\right)(a=1,2)$ in $\left(C^{0}(M, N) \times C^{0}\left(\Sigma M \otimes \phi_{a}^{-1} T N\right)\right) \bigcap\left(C^{\infty}(\stackrel{\circ}{M}, N) \times\right.$ $\left.C^{\infty}\left(\Sigma \stackrel{\circ}{M} \otimes \phi_{a}^{-1} T N\right)\right)$ satisfying

$$
\phi_{a}(M) \subset B_{R}\left(y_{0}\right), \quad\left|\psi_{a}\right|^{2}<C_{0}, \quad a=1,2,
$$

the maximum principle holds, namely

$$
\sup _{M} \Theta \leq \sup _{\partial M} \Theta
$$

Furthermore, assume that $\left(\phi_{a}, \psi_{a}\right) \in\left(H^{2, p}(M, N) \times H^{1, p}\left(\Sigma M \otimes \phi_{a}^{-1} T N\right)\right) \bigcap\left(C^{\infty}\right.$ $\left.(\stackrel{\circ}{M}, N) \times C^{\infty}\left(\Sigma \stackrel{\circ}{M} \otimes \phi_{a}^{-1} T N\right)\right), p>n=\operatorname{dim} M$, we have

$$
\begin{aligned}
\left\|\phi_{1}-\phi_{2}\right\|_{C^{0}(M)}+ & \left\|\psi_{1}-\psi_{2}\right\|_{C^{0}(M)} \\
& \leq C\left(\left\|\phi_{1}-\phi_{2}\right\|_{H^{2-\frac{1}{p}, p}(\partial M)}+\left\|\mathbf{B} \psi_{1}-\mathbf{B} \psi_{2}\right\|_{H^{1-\frac{1}{p}, p}(\partial M)}\right)
\end{aligned}
$$

for some constant $C=C\left(n, n^{\prime}, p, y_{0}, R, C_{0}, M, N\right)>0$. In particular, if $\left.\phi_{1}\right|_{\partial M}=\left.\phi_{2}\right|_{\partial M}$, and $\mathbf{B} \psi_{1}=\mathbf{B} \psi_{2}$, then

$$
\left(\phi_{1}, \psi_{1}\right) \equiv\left(\phi_{2}, \psi_{2}\right)
$$

Here, the boundary operator $\mathbf{B}$ is defined by (2.31) below.

We will also prove the following existence theorem for a boundary value problem of Dirac-harmonic maps. As is known from harmonic maps, we need to impose a smallness condition on the map here, in order to guarantee that its image be contained in a convex ball. Analogously, we need a smallness condition on the spinor field, as explained above. Thus, what we achieve here is a local existence theorem from the perspective of geometry.

Theorem 1.2 (Existence theorem for Dirac-harmonic maps). Let $M, N, y_{0}, \kappa, R$ and $C_{0}$ be as in Theorem 1.1. Then there exist positive constants $a_{0}$ and $b_{0}$ such that for any $\phi_{0} \in H^{2, p}(M, N)$ with $\left\|\phi_{0}\right\|_{H^{2, p}(M)}<a_{0}$ and any $\psi_{0} \in H^{1, p}\left(\Sigma M \otimes \phi_{0}^{-1}(T N)\right)$ with $\left\|\psi_{0}\right\|_{H^{1, p}(M)}<b_{0}$, where $p>n:=\operatorname{dim} M$, the boundary value problem

$$
\begin{aligned}
& \left\{\begin{array}{l}
\tau(\phi)=\mathcal{R}(\phi, \psi), \\
D \psi=0,
\end{array}\right. \\
& \left\{\begin{array}{l}
\left.\phi\right|_{\partial M}=\left.\phi_{0}\right|_{\partial M}, \\
\mathbf{B} \psi=\mathbf{B} \psi_{0}
\end{array}\right.
\end{aligned}
$$


admits a unique solution $(\phi, \psi) \in H^{2, p}(M, N) \times H^{1, p}\left(\Sigma M \otimes \phi^{-1}(T N)\right)$ satisfying $\phi(M) \subset$ $B_{R}\left(y_{0}\right)$ and $|\psi|^{2}<C_{0}$.

Remark 1.1 (i) In Theorem 1.1 the condition that the scalar curvature of $M$ is positive cannot be removed for the uniqueness. Without it, uniqueness does not hold even for harmonic spinor fields. The scalar curvature comes in through the Lichnerowicz formula that we need to apply to the spinor fields $\psi_{1}, \psi_{2}$. When the scalar curvature is positive, this will yield a positive contribution that can be used to absorb a term involving $|\psi|^{4}$ when we assume in addition that that norm be sufficiently small. For details, see the summary of our estimates in (3.28) below.

(ii) We expect that Theorem 1.1 and Theorem 1.2 hold for any fixed $R<\frac{\pi}{2 \sqrt{\kappa}}$ and $C_{0}<\infty$. Similarly, when $\kappa=0$, that is, for targets of nonpositive sectional curvature, we expect that Theorem 1.1 and Theorem 1.2 hold for any fixed $R<\infty$ and $C_{0}<\infty$.

This paper is organized as follows. In Sect. 2, we give some preliminary results on pseudo distances of vectors and global estimates for Dirac operators with elliptic boundary conditions, and we define the chirality boundary condition for Dirac-harmonic maps considered in this paper. In Sect. 3, we prove the maximum principle and the uniqueness (Theorem 1.1) for Dirac-harmonic maps from manifolds with boundary into small regular balls. In Sect. 4, we prove the existence theorem (Theorem 1.2) for chirality boundary value problems of Dirac-harmonic maps into small regular balls.

\section{Pseudo distances of vectors and global estimates for Dirac operator with elliptic boundary conditions}

In this section, we will recall two notions of pseudo distances $\delta\left(v_{1}, v_{2}\right), \delta_{0}\left(v_{1}, v_{2}\right)$ between two vectors $v_{1}, v_{2}$ on $N$ and derive a relation between them. We will also consider global estimates for the usual Dirac operator $\not \partial$ on $M$ with elliptic boundary conditions. These will be used in the next sections; they are also of interest in their own right.

\subsection{Pseudo distances}

For any $y_{1}, y_{2} \in B_{R}\left(y_{0}\right)$, there exists a unique minimal geodesic $\sigma:[0, \rho] \rightarrow B_{R}\left(y_{0}\right) \subset N$ such that $\sigma(0)=y_{1}, \sigma(\rho)=y_{2}$, where $\rho:=\operatorname{dist}\left(y_{1}, y_{2}\right)$ stands for the distance of $y_{1}, y_{2}$ on $N$. For any $v_{a} \in T_{y_{a}} N, a=1,2$, let $X$ be the unique Jacobi field along $\sigma$ with $X(0)=$ $v_{1}, X(\rho)=v_{2}$.

We start by defining (pseudo-)distances between vector fields or linear maps (c.f. [18]).

Definition 2.1 For a pair $v_{a} \in T_{y_{a}} N$ of tangent vectors, $a=1$, 2, we define a pseudo-distance

$$
\delta\left(v_{1}, v_{2}\right):=\left\{\begin{array}{l}
\left(\rho \int_{0}^{\rho}|\dot{X}|^{2}\right)^{\frac{1}{2}} \text { if } \rho>0, \\
\left|v_{1}-v_{2}\right|, \quad \text { if } \rho=0 .
\end{array}\right.
$$

For $p, q \in \bigsqcup_{y \in B_{R}\left(y_{0}\right)} P_{x, y}$ (disjoint union), we then define their pseudo-distance as

$$
\delta(p, q):=\sum_{\alpha=1}^{n} \delta^{2}\left(p\left(e_{\alpha}\right), q\left(e_{\alpha}\right)\right)^{1 / 2},
$$

where $\left\{e_{1}, \cdots, e_{n}\right\}$ is an orthonormal base in $T_{x} M$. 
We note that $\delta\left(v_{1}, v_{2}\right)=0$ if the tangent vector $v_{2}$ is obtained from $v_{1}$ by parallel transport along the shortest geodesic between their base points. Therefore, $\delta$ is not a true distance.

This notion of a pseudo-distance arises from the Hessian of the distance function $\operatorname{dist}(\cdot, \cdot)$ on $N \times N$. Recall that when $v_{1}$ and $v_{2}$ are normal to $\sigma$, for $v:=v_{1} \oplus v_{2} \in T_{\left(y_{1}, y_{2}\right)}(N \times N)$, we have

$$
\left(\nabla^{2} \text { dist }\right)(v, v)=\int_{0}^{\rho}\left(|\dot{X}|^{2}-\left\langle X, R\left(X, \sigma^{\prime}\right) \sigma^{\prime}\right\rangle\right) .
$$

The first term on the RHS is $\rho^{-1} \delta^{2}\left(v_{1}, v_{2}\right)$. This makes the pseudo distance $\delta(\cdot, \cdot)$ important in the estimates of the Laplacian of distance functions between two maps $\phi_{1}, \phi_{2}: M \rightarrow N$. However, in applications, one often encounters another kind of pseudo distance function defined as follows:

$$
\delta_{0}\left(v_{1}, v_{2}\right):=\left|v_{1}-\overline{\bar{v}}_{2}\right|,
$$

where $\overline{\bar{v}}_{2}$ stands for the vector in $T_{y_{1}} N$ obtained by the parallel displacement of $v_{2}$ along $\sigma$. This one is geometrically more natural and convenient to use. However, the maximum principle of Jäger-Kaul [18] is formulated in terms of $\delta$, and not of $\delta_{0}$, and it turns out the precise properties of $\delta$ are really needed. Essentially, the reason is that parallel transport can be estimated as precisely as Jacobi fields in terms of curvature conditions, because the latter satisfy a differential equation. In particular, the estimate (2.19) below will be crucial.

Nevertheless, the following relation between the above two pseudo distances will be useful. Since $\delta$ is expressed in terms of the derivative of a Jacobi field connecting the two vectors in question, it is natural that estimates for Jacobi fields (c.f. [21], [26]) can be utilized to control $\delta$ and $\delta_{0}$ in terms of each other.

Lemma 2.1 There is a positive constant $C$ depending only on $B_{R}\left(y_{0}\right)$ and the geometry of $N$ such that for any $y_{a} \in B_{R}\left(y_{0}\right)$ and $v_{a} \in T_{y_{a}} N, a=1,2$, we have

$$
\begin{aligned}
\delta_{0}^{2}\left(v_{1}, v_{2}\right)-C\left(\left|v_{1}\right|^{2}+\left|v_{2}\right|^{2}\right) \rho^{2} & \leq \delta^{2}\left(v_{1}, v_{2}\right) \\
& \leq \delta_{0}^{2}\left(v_{1}, v_{2}\right)+C\left(\left|v_{1}\right|^{2}+\left|v_{2}\right|^{2}\right) \rho^{2} .
\end{aligned}
$$

Proof First, it is easy to verify that for both pseudo distances $\delta$ and $\delta_{0}$, the Pythagorean law holds true:

$$
\begin{aligned}
& \delta^{2}\left(v_{1}, v_{2}\right)=\delta^{2}\left(v_{1}^{\tan }, v_{2}^{\tan }\right)+\delta^{2}\left(v_{1}^{\text {nor }}, v_{2}^{\text {nor }}\right), \\
& \delta_{0}^{2}\left(v_{1}, v_{2}\right)=\delta_{0}^{2}\left(v_{1}^{\text {tan }}, v_{2}^{\tan }\right)+\delta_{0}^{2}\left(v_{1}^{\text {nor }}, v_{2}^{\text {nor }}\right) .
\end{aligned}
$$

Clearly, $\delta\left(v_{1}^{\tan }, v_{2}^{\tan }\right)=\delta_{0}\left(v_{1}^{\tan }, v_{2}^{\tan }\right)=\left|\left\langle\sigma^{\prime}(\rho), v_{2}\right\rangle-\left\langle\sigma^{\prime}(0), v_{1}\right\rangle\right|$, hence, in the following, we may assume that $v_{1}$ and $v_{2}$ are normal to the geodesic $\sigma$.

Let $X$ be the unique Jacobi field along $\sigma$ with boundary values

$$
X(0)=v_{1}, \quad X(\rho)=v_{2} .
$$

Define a Jacobi field $Y(\cdot)$ along $\sigma$ such that

$$
Y(0)=v_{1}, \quad Y(\rho)=\overline{\bar{v}}_{1} .
$$

Set $Z(t):=X(t)-Y(t), \forall t \in[0, \rho]$, then $Z$ is a Jacobi field along $\sigma$ with

$$
Z(0)=0, \quad Z(\rho)=v_{2}-\overline{\bar{v}}_{1} .
$$


To estimate $\delta^{2}\left(v_{1}, v_{2}\right) \equiv \rho \int_{0}^{\rho}\left|X^{\prime}\right|^{2}$, we need to estimate $\left|Y^{\prime}\right|$ and $\left|Z^{\prime}\right|$.

The Jacobi field $Z$ can be written as:

$$
Z(t)=\left(d \exp _{y_{1}}\right)_{t \sigma^{\prime}(0)}\left(t Z^{\prime}(0)\right) .
$$

Assume that the sectional curvature of $N$ satisfies

$$
\theta \leq K_{N} \leq \kappa
$$

where $\theta<0$ is a constant. Denote

$$
S_{\kappa}(t):=\frac{1}{\sqrt{\kappa}} \sin \sqrt{\kappa} t, \quad S_{\theta}(t):=\frac{1}{\sqrt{-\theta}} \sinh \sqrt{-\theta} t .
$$

Then for any vector $w \in T_{y_{1}} N$, we have (c.f. Corollary 4.6.1 in [21]):

$$
|w| S_{\kappa}(t) \leq\left|\left(d \exp _{y_{1}}\right)_{t \sigma^{\prime}(0)}(t w)\right| \leq|w| S_{\theta}(t) .
$$

Putting $w=Z^{\prime}(0)$ and $Z(\rho)=v_{2}-\overline{\bar{v}}_{1}$ into these inequalities then yields

$$
\left|Z^{\prime}(0)\right| \frac{1}{\sqrt{\kappa}} \sin \sqrt{\kappa} \rho \leq\left|v_{2}-\overline{\bar{v}}_{1}\right| \leq\left|Z^{\prime}(0)\right| \frac{1}{\sqrt{-\theta}} \sinh \sqrt{-\theta} \rho,
$$

namely,

$$
\frac{\sqrt{-\theta}\left|v_{2}-\overline{\bar{v}}_{1}\right|}{\sinh \sqrt{-\theta} \rho} \leq \mu:=\left|Z^{\prime}(0)\right| \leq \frac{\sqrt{\kappa}\left|v_{2}-\overline{\bar{v}}_{1}\right|}{\sin \sqrt{\kappa} \rho} .
$$

Estimate of $\left|Y^{\prime}\right|$.

Let $J(\cdot)$ be the unique Jacobi field along $\sigma$ such that

$$
J(0)=v_{1}, \quad J^{\prime}(0)=0 .
$$

Choose a parallel orthonormal frames $\left\{E_{i}(t)\right\}$ along $\sigma$ with $E_{1}=\sigma^{\prime}$, and denote

$$
f_{i}(t):=\left\langle J(t), E_{i}(t)\right\rangle, \quad i=2, \cdots, n^{\prime},
$$

then we have the following expansions:

$$
f_{i}(t)=v_{1}^{i}-\frac{t^{2}}{2}\left\langle R_{\sigma^{\prime} v_{1}} \sigma^{\prime}, E_{i}\right\rangle(0)+O\left(t^{3}\right), \quad i=2, \cdots, n^{\prime},
$$

consequently,

$$
J(t)=V_{1}(t)+\left|v_{1}\right| O\left(t^{2}\right),
$$

where $V_{1}$ is the vector field obtained by the parallel displacement of $v_{1}$ along $\sigma$.

Define $\widetilde{Y}(t):=Y(t)-J(t)$, it is a Jacobi field along $\sigma$ with boundary values

$$
\tilde{Y}(0)=0, \quad \tilde{Y}(\rho)=\overline{\bar{v}}_{1}-J(\rho)=\left|v_{1}\right| O\left(\rho^{2}\right) .
$$

By rescaling, we obtain a Jacobi field

$$
\bar{Y}(s):=\frac{1}{\rho^{2}} \tilde{Y}(\rho s), \quad \forall s \in[0,1]
$$

along the geodesic $\bar{\sigma}(s):=\sigma(\rho s), s \in[0,1]$. The boundary values of $\bar{Y}$ are

$$
\bar{Y}(0)=0, \quad \bar{Y}(1)=\left|v_{1}\right| O(1),
$$


by the ODE theory, it is easy to see that there is a positive constant $C$ such that $\left|\bar{Y}^{\prime}\right| \leq C\left|v_{1}\right|$. Scaling back, we have $\left|\widetilde{Y}^{\prime}\right| \leq C\left|v_{1}\right| \rho$, therefore,

$$
\left|Y^{\prime}\right|=\left|\tilde{Y}^{\prime}+J^{\prime}\right|=\left|v_{1}\right| O(\rho)
$$

and consequently,

$$
\rho \int_{0}^{\rho}\left|Y^{\prime}(t)\right|^{2}=\left|v_{1}\right|^{2} O\left(\rho^{4}\right) .
$$

Estimate of $\left|Z^{\prime}\right|$.

Set $h_{i}(t):=\left\langle Z^{\prime}(t), E_{i}(t)\right\rangle, i=1,2, \cdots, n^{\prime}$, then

$$
h_{i}^{\prime}(t)=\left\langle Z^{\prime \prime}(t), E_{i}(t)\right\rangle=-\left\langle R_{\sigma^{\prime} Z^{\sigma^{\prime}}}, E_{i}\right\rangle(t)=-|Z|\left\langle R_{\sigma^{\prime} \frac{Z}{|Z|}} \sigma^{\prime}, E_{i}\right\rangle(t),
$$

hence,

$$
h_{i}(t)-h_{i}(0)=t h_{i}^{\prime}\left(\theta_{i}\right)=-t|Z|\left\langle R_{\sigma^{\prime} \frac{Z}{|Z|}} \sigma^{\prime}, E_{i}\right\rangle\left(\theta_{i}\right)
$$

for some $\theta_{i} \in[0, \rho], i=1,2, \cdots, n^{\prime}$. Thus, we have

$$
\begin{aligned}
Z^{\prime}(t) & =h_{i}(0) E_{i}(t)-t|Z|\left\langle R_{\sigma^{\prime} \frac{Z}{|Z|}} \sigma^{\prime}, E_{i}\right\rangle\left(\theta_{i}\right) E_{i}(t) \\
& :=P_{Z^{\prime}(0)}(t)-t|Z| A(t),
\end{aligned}
$$

where $P_{Z^{\prime}(0)}(t)$ is the vector field obtained by the parallel displacement of $Z^{\prime}(0)$ along $\sigma$, and $|A(t)|=\left|\left\langle R_{\sigma^{\prime} \frac{Z}{|Z|}} \sigma^{\prime}, E_{i}\right\rangle\left(\theta_{i}\right) E_{i}(t)\right| \leq C$ for some constant $C>0$.

Noting that $\left|P_{Z^{\prime}(0)}(t)\right|=\left|Z^{\prime}(0)\right|=\mu, \forall t \in[0, \rho]$, we have

$$
\left|Z^{\prime}(t)\right|^{2}=\left|Z^{\prime}(0)\right|^{2}+t^{2}|Z|^{2} A^{2}(t)-2 t|Z|\left\langle P_{Z^{\prime}(0)}(t), A(t)\right\rangle .
$$

Recalling that by (A4) in [26], we have

$$
|Z(t)| \leq f_{\theta}(t)
$$

for $t \in[0, \rho]$ and $\rho$ small, where $f_{\theta}(t):=\frac{\mu}{\sqrt{-\theta}} \sinh \sqrt{-\theta} t=\mu S_{\theta}(t)$. Substituting this into (2.9) we have

$$
\mu^{2}-C \mu^{2} t S_{\theta}(t)-C \mu^{2} t^{2} S_{\theta}^{2}(t) \leq\left|Z^{\prime}(t)\right|^{2} \leq \mu^{2}+C \mu^{2} t S_{\theta}(t)+C \mu^{2} t^{2} S_{\theta}^{2}(t) .
$$

Consequently, we have

$$
\begin{aligned}
\rho \int_{0}^{\rho}\left|Z^{\prime}(t)\right|^{2} d t & \leq \mu^{2} \rho^{2}\left(1+O\left(\rho^{2}\right)\right) \\
& \leq \rho^{2} \frac{\kappa\left|v_{2}-\overline{\bar{v}}_{1}\right|^{2}}{\sin ^{2} \sqrt{\kappa} \rho}\left(1+O\left(\rho^{2}\right)\right) \quad \text { by } \\
& =\delta_{0}^{2}\left(v_{1}, v_{2}\right)\left(1+O\left(\rho^{2}\right)\right)
\end{aligned}
$$


Note that

$$
\begin{aligned}
\delta^{2}\left(v_{1}, v_{2}\right) & =\rho \int_{0}^{\rho}\left|Z^{\prime}+Y^{\prime}\right|^{2} \\
& \leq \rho \int_{0}^{\rho}\left|Z^{\prime}\right|^{2}+\rho \int_{0}^{\rho}\left|Y^{\prime}\right|^{2}+2 \rho \int_{0}^{\rho}\left|Z^{\prime}\right|\left|Y^{\prime}\right|,
\end{aligned}
$$

combining this with (2.11), (2.8), (2.5), (2.10) and (2.7) we obtain

$$
\delta^{2}\left(v_{1}, v_{2}\right) \leq \delta_{0}^{2}\left(v_{1}, v_{2}\right)+C\left(\left|v_{1}\right|^{2}+\left|v_{2}\right|^{2}\right) \rho^{2} .
$$

Similarly, we can deduce

$$
\delta^{2}\left(v_{1}, v_{2}\right) \geq \delta_{0}^{2}\left(v_{1}, v_{2}\right)-C\left(\left|v_{1}\right|^{2}+\left|v_{2}\right|^{2}\right) \rho^{2} .
$$

This proves Lemma 2.1 .

Corollary 1 There exist positive constants $C, C^{\prime}$ depending only on $B_{R}\left(y_{0}\right)$ and the geometry of $N$ such that for any $\phi_{1}, \phi_{2}: M \rightarrow B_{R}\left(y_{0}\right)$, the following estimates hold:

$$
\begin{aligned}
& C^{\prime}\left|d \phi_{1}-d \phi_{2}\right|^{2}-C \rho^{2} \sum_{a=1}^{2}\left|d \phi_{a}\right|^{2} \leq \delta_{0}^{2}\left(d \phi_{1}, d \phi_{2}\right) \leq C\left|d \phi_{1}-d \phi_{2}\right|^{2}+C \rho^{2} \sum_{a=1}^{2}\left|d \phi_{a}\right|^{2}, \\
& C^{\prime}\left|d \phi_{1}-d \phi_{2}\right|^{2}-C \rho^{2} \sum_{a=1}^{2}\left|d \phi_{a}\right|^{2} \leq \delta^{2}\left(d \phi_{1}, d \phi_{2}\right) \leq C\left|d \phi_{1}-d \phi_{2}\right|^{2}+C \rho^{2} \sum_{a=1}^{2}\left|d \phi_{a}\right|^{2} .
\end{aligned}
$$

Proof For any $x \in M$, let $\sigma$ be the unique geodesic connecting $\phi_{1}(x)$ and $\phi_{2}(x)$, choose a parallel orthonormal frame $\left\{E_{i}(t)\right\}$ along $\sigma$ with $E_{1}=\sigma^{\prime}$, and a local orthonormal frame $\left\{e_{\alpha}\right\}_{\alpha=1,2, \cdots, n}$ around $x$ in $M$. Assume $\partial_{y^{i}}:=a_{i}^{j} E_{j}$, and denote $\phi_{1 \alpha}:=d \phi_{1}\left(e_{\alpha}\right):=$ $\phi_{1 \alpha}^{k} \partial_{y^{k}}\left(\phi_{1}\right)$ and $\phi_{2 \alpha}:=d \phi_{2}\left(e_{\alpha}\right):=\phi_{2 \alpha}^{k} \partial_{y^{k}}\left(\phi_{2}\right)$, then

$$
\begin{aligned}
\delta_{0}\left(\phi_{1 \alpha}, \phi_{2 \alpha}\right) & =\delta_{0}\left(\phi_{1 \alpha}^{i} \partial_{y^{i}}\left(\phi_{1}\right), \phi_{2 \alpha}^{i} \partial_{y^{i}}\left(\phi_{2}\right)\right) \\
& =\delta_{0}\left(\phi_{1 \alpha}^{i} a_{i}^{j}\left(\phi_{1}\right) E_{j}\left(\phi_{1}\right), \phi_{2 \alpha}^{i} a_{i}^{j}\left(\phi_{2}\right) E_{j}\left(\phi_{2}\right)\right),
\end{aligned}
$$

by definition of the pseudo distance $\delta_{0}$, we have

$$
\begin{aligned}
\delta_{0}^{2}\left(\phi_{1 \alpha}, \phi_{2 \alpha}\right) & =\sum_{j}\left(\phi_{1 \alpha}^{i} a_{i}^{j}\left(\phi_{1}\right)-\phi_{2 \alpha}^{i} a_{i}^{j}\left(\phi_{2}\right)\right)^{2} \\
& =\sum_{j}\left[\left(\phi_{1 \alpha}^{i}-\phi_{2 \alpha}^{i}\right) a_{i}^{j}\left(\phi_{1}\right)+\phi_{2 \alpha}^{i}\left(a_{i}^{j}\left(\phi_{1}\right)-a_{i}^{j}\left(\phi_{2}\right)\right)\right]^{2} \\
& \leq C \sum_{j}\left(\phi_{1 \alpha}^{j}-\phi_{2 \alpha}^{j}\right)^{2}+C \rho^{2} \sum_{a=1}^{2}\left|d \phi_{a}\right|^{2}
\end{aligned}
$$


where $C>0$ is a constant depending on the bounds of $\left(a_{j}^{i}\right)$ and $\left(d a_{j}^{i}\right)$ on $B_{R}\left(y_{0}\right)$, and we have used the fact that $h_{i j}=a_{i}^{k} a_{j}^{k}$ and $h_{i j}\left(y_{0}\right)=\delta_{i j}$. Similarly, we can show that

$$
\delta_{0}^{2}\left(\phi_{1 \alpha}, \phi_{2 \alpha}\right) \geq C^{\prime} \sum_{j}\left(\phi_{1 \alpha}^{j}-\phi_{2 \alpha}^{j}\right)^{2}-C \rho^{2} \sum_{a=1}^{2}\left|d \phi_{a}\right|^{2} .
$$

Then (2.13) follows from (2.16) and (2.17). From Lemma 2.1 and (2.13), we immediately have (2.14).

The following lemma is from [18] (Lemma 3.16). The main point is that it estimates the Hessian of the distance function from below in terms of the pseudo distance $\delta$. In other words, it provides a precise quantitative version of the convexity properties of the distance function of the image. This will be crucial below when this distance function is composed with Dirac-harmonic maps, in order to obtain a useful differential inequality that will be at the heart of the maximum principle.

Lemma 2.2 (1) Denote $G(\cdot):=q_{\kappa} \circ \operatorname{dist}\left(y_{0}, \cdot\right): B_{R}\left(y_{0}\right) \rightarrow \mathbb{R}$, then

$$
\nabla^{2} G(u, u) \geq s_{\kappa}^{\prime}(\tau)|u|^{2}
$$

$\forall u \in T_{x} N, x \in B_{R}\left(y_{0}\right), \tau:=\operatorname{dist}\left(y_{0}, x\right)$.

(2) Denote $F:=q_{\frac{\kappa}{4}} \circ$ dist $: B_{R}\left(y_{0}\right) \times B_{R}\left(y_{0}\right) \rightarrow \mathbb{R}$, then

$$
\nabla^{2} F(v, v) \geq s_{\frac{\kappa}{4}}^{\prime}(\rho) \delta^{2}\left(v_{1}, v_{2}\right)-s_{\frac{\kappa}{4}}(\rho) b_{\kappa}(\rho) \sum_{a=1}^{2}\left|v_{a}\right|^{2},
$$

$$
\forall v=v_{1} \otimes v_{2}, v_{a} \in T_{y_{a}} N, y_{a} \in B_{R}\left(y_{0}\right), a=1,2, \text { and }
$$

$$
\begin{aligned}
& b_{\kappa}(t):= \begin{cases}\frac{1}{2} a_{\kappa}(t)\left(1+\frac{t}{s_{\kappa}(t)}\right) & \text { if } t>0, \\
0 & \text { if } t=0,\end{cases} \\
& a_{\kappa}(t):= \begin{cases}s_{\kappa}^{-1}(t)\left(1-s_{\kappa}^{\prime}(t)\right) & \text { if } t>0, \\
0 & \text { if } t=0 .\end{cases}
\end{aligned}
$$

2.2 Global elliptic estimates for the Dirac operator

In this subsection, we shall recall the boundary condition for the usual Dirac operator and extend it to Dirac operators along maps. We shall then consider global elliptic estimates for the Dirac operator with boundary conditions. These will be important for us to deal with the spinor field $\psi$ of a Dirac-harmonic map $(\phi, \psi)$.

Let $M$ be an $n$-dimensional compact Riemannian spin manifold with boundary $\partial M \neq \emptyset$. First, we recall some notions and facts about the spin bundles and the usual Dirac operators on $\partial M$ (see e.g. [4], [6] and [14]).

Let $\mathbf{S}:=\left.\Sigma M\right|_{\partial M}$ be the restricted spinor bundle with induced Hermitian product. Then

$$
\mathbf{S} \cong\left\{\begin{array}{lll}
\mathbf{S} \partial M, & n & \text { odd } \\
\mathbf{S} \partial M \oplus \mathbf{S} \partial M, & n & \text { even }
\end{array}\right.
$$


Define the Clifford multiplication and covariant derivative on $\mathbf{S}$ by

$$
\gamma^{\mathbf{S}}(X) \Psi:=\gamma(X) \gamma(\nu) \Psi \quad \text { and } \quad \nabla_{X}^{\mathbf{S}} \Psi:=\nabla_{X} \Psi-\frac{1}{2} \gamma \mathbf{S}_{(A X) \Psi}
$$

respectively, $\forall X \in \Gamma(T \partial M), \Psi \in \Gamma(\mathbf{S})$ on $\partial M$, where $v$ is the unit normal vector field of $\partial M, A$ denotes the shape operator of $\partial M$ in $M$, and $\gamma(\cdot)$ stands for the Clifford multiplication on $\Sigma M$.

Let $u_{1}, u_{2}, \cdots, u_{n-1}$ be a local orthonormal frame on $\partial M$. Then the hypersurface Dirac operator $\mathbf{D}$ is defined as

$$
\mathbf{D} \Psi:=\sum_{j=1}^{n-1} \gamma^{\mathbf{S}}\left(u_{j}\right) \nabla_{u_{j}}^{\mathbf{S}} \Psi=\frac{n-1}{2} H \Psi-\gamma(v) \sum_{j=1}^{n-1} \gamma\left(u_{j}\right) \nabla_{u_{j}} \Psi, \quad \forall \Psi \in \Gamma(\mathbf{S}),
$$

where $H$ is the mean curvature of $\partial M$ in $M$.

Let us now impose the boundary condition for the spinor field $\psi$. There are various types of boundary conditions in the physics and mathematics literature-the APS condition, the chirality condition, the Riemannian version of the MIT bag condition, the mAPS condition etc. - which are elliptic for the usual Dirac operator (see [1-3], [5], [7], [12], [14], [17]).

Throughout this paper, our boundary conditions for Dirac operators will refer to either of the above mentioned four conditions. By the exactly same arguments, our conclusions in Theorems 1.1 and 1.2 concerning the boundary conditions hold for all those four conditions. Here we take the example of the chirality condition. This is a local boundary condition, we will extend it to our settings (see [10] for the free boundary condition of Dirac-harmonic maps).

Definition 2.2 A chirality operator $G$ is an endomorphism of the spinor bundle $\Sigma M$ satisfying:

$$
\begin{gathered}
G^{2}=I, \quad\langle G \psi, G \varphi\rangle=\langle\psi, \varphi\rangle, \\
\nabla_{X}(G \psi)=G \nabla_{X} \psi, \quad \gamma(X) G \psi=-G(\gamma(X) \psi) .
\end{gathered}
$$

$\forall X \in \Gamma(T M), \psi, \varphi \in \Gamma(\Sigma M)$. Here $I$ denotes the identity endomorphism of $\Sigma M$.

When the dimension $n$ of $M$ is even, we have the usual chirality operator $G=\gamma\left(\omega_{n}\right)$, the Clifford multiplication by the complex volume form $\omega_{n}$.

Suppose that $M$ admits a chirality operator $G$. One can verify that:

$$
\langle\gamma(v) G \psi, \varphi\rangle=\langle\psi, \gamma(v) G \varphi\rangle, \quad(\gamma(v) G)^{2}=I .
$$

This allows us to decompose $\mathbf{S}=V^{+} \oplus V^{-}$, where $V^{ \pm}$is the eigensubbundle corresponding to the eigenvalue \pm 1 . Correspondingly, we have the orthogonal projections onto the eigensubbundles $V^{ \pm}$:

$$
\begin{aligned}
\mathbf{B}^{ \pm}: L^{2}(\mathbf{S}) & \rightarrow L^{2}\left(V^{ \pm}\right) \\
\psi & \mapsto \frac{1}{2}(I \pm \gamma(v) G) \psi .
\end{aligned}
$$

Based on this, one can define a local elliptic boundary condition-the chirality condition - for the Dirac operator $\not \partial$ (see e.g. [12], [14]) as follows: for $\psi \in \Gamma(\Sigma M)$ and a given spinor field $b_{0} \in L^{2}\left(V^{-}\right)$,

$$
\mathbf{B} \psi=b_{0},
$$


where

$$
\mathbf{B} \psi:=\left.\mathbf{B}^{-} \psi\right|_{\partial M}:=\left.\frac{1}{2}(I-\gamma(v) G) \psi\right|_{\partial M} .
$$

For a given $\psi_{0} \in \Gamma(\Sigma M)$, we can then impose the boundary condition

$$
\mathbf{B} \psi=\mathbf{B} \psi_{0} .
$$

The next lemma tells us that the Sobolev norm of $\psi \in \Gamma(\Sigma M)$ can be controlled in terms of the norm of its Dirac and its boundary condition. Of course, this is essentially an ellipticity argument.

Lemma 2.3 Let $M$ be a compact Riemannian spin manifold with boundary $\partial M$ which has non-negative mean curvature (w.r.t. the inner normal), assume that the scalar curvature of $M$ satisfies $\inf _{M} S_{M}>0$. Let $\mathbf{B}$ be a boundary condition for the Dirac operator $p$. Then

$$
\|\psi\|_{H^{1, p}(\Sigma M)} \leq C\left(\|\not \partial \psi\|_{L^{p}(\Sigma M)}+\|\mathbf{B} \psi\|_{H^{1-\frac{1}{p}, p}(\mathbf{S})}\right), \quad \forall p>1, \quad \forall \psi \in \Gamma(\Sigma M)
$$

where $C=C(p, M)>0$ is a constant.

Proof Consider the following operator between Banach spaces:

$$
(\not \partial, \mathbf{B}): H^{1, p}(\Sigma M) \rightarrow L^{p}(\Sigma M) \oplus H^{1-\frac{1}{p}, p}(\mathbf{S}) .
$$

For any $\Psi$ in the kernel $\operatorname{Ker}(\not \partial, \mathbf{B})$, by the definitions of $\mathbf{B}, \mathbf{D}$ and $G$, it is easy to verify that (c.f. [14]) on the boundary $\partial M$

$$
\int_{\partial M}\langle\mathbf{D} \Psi, \Psi\rangle \leq 0
$$

Recall that we have the following spinorial Reilly inequality (see e.g. [14]):

$$
\int_{\partial M}\left[\langle\mathbf{D} \Psi, \Psi\rangle-\frac{n-1}{2} H|\Psi|^{2}\right] \geq \int_{M}\left[\frac{S_{M}}{4}|\Psi|^{2}-\frac{n-1}{n}|\partial \Psi|^{2}\right], \quad \forall \Psi \in \Gamma(\Sigma M) .
$$

Using this we have

$$
0 \geq \int_{M} \frac{S_{M}}{4}|\Psi|^{2}+\int_{\partial M} \frac{n-1}{2} H|\Psi|^{2}, \quad \forall \Psi \in \Gamma(\Sigma M) .
$$

From our assumptions it then follows that $\Psi \equiv 0$. Namely, $\operatorname{Ker}(\not \partial, \mathbf{B})=\{0\}$.

Since the boundary condition is elliptic, the operator $(\not \partial, \mathbf{B})$ is Fredholm and therefore (c.f. [28], Proposition 1.5.8),

$$
\|\psi\|_{H^{1, p}(\Sigma M)} \leq C\left(\|\not \partial \psi\|_{L^{p}(\Sigma M)}+\|\mathbf{B} \psi\|_{H^{1-\frac{1}{p}, p}(\mathbf{S})}\right)
$$

for some constant $C(p, M)>0$.

Finally, we extend the above boundary condition to Dirac operators along maps. 
Definition 2.3 For any spinor field $\psi \in \Gamma\left(\Sigma M \otimes \phi^{-1} T N\right)$ along a map $\phi$, if $\psi:=\psi^{i} \otimes$ $\frac{\partial}{\partial y^{i}}(\phi)$ in local coordinates $\left(y^{i}\right)_{i=1,2, \cdots, n^{\prime}}$ in $N$, we define

$$
\mathbf{B} \psi:=\left(\mathbf{B} \psi^{i}\right) \otimes \frac{\partial}{\partial y^{i}} .
$$

For a given spinor field $\psi_{0} \in \Gamma\left(\Sigma M \otimes \phi_{0}^{-1} T N\right)$ along a map $\phi_{0}$ satisfying $\left.\phi\right|_{\partial M}=\left.\phi_{0}\right|_{\partial M}$, we define the chirality boundary condition:

$$
\mathbf{B} \psi=\mathbf{B} \psi_{0} .
$$

Remark 2.1 Clearly, (2.31) is independent of the choice of local coordinates.

\section{Maximum principle and uniqueness for Dirac-harmonic maps}

In this section, we will prove the maximum principle and uniqueness for Dirac-harmonic maps from manifolds with boundary. The main idea to prove a maximum principle for maps between manifolds is to construct an appropriate elliptic operator $L$ and apply it on some function $\Theta$ of the distance of two maps, such that $L(\Theta) \geq 0$. A natural choice of $L$ is the Laplacian operator $\Delta$. However, $\Delta \Theta$ usually contains some negative terms which prevent $\Delta \Theta$ from being nonnegative. Therefore, one needs to modify the operator $\Delta$ as well as the function $\Theta$ to obtain an operator $L$ and a new $\Theta$ that create positive terms to cancel out those negative terms. A clever construction of $L$ and $\Theta$ was given in [18] and [19]. This construction motivates our proof below, but we need to work harder in order to handle the Dirac term.

Proof of Theorem 1.1. For a pair of Dirac-harmonic maps $\left(\phi_{1}, \psi_{1}\right),\left(\phi_{2}, \psi_{2}\right)$ into the regular ball $B_{R}\left(y_{0}\right)$, we introduce the following function of distances:

$$
\widetilde{\xi}:=q_{\frac{\kappa}{4}} \circ \operatorname{dist}\left(\phi_{1}, \phi_{2}\right)+\frac{1}{2}\left|\psi_{1}-\psi_{2}\right|^{2},
$$

Define an elliptic operator $\mathfrak{L}$ as follows:

$$
\mathfrak{L}(f):=e^{\Phi} \operatorname{div}\left(e^{-2 \Phi} \nabla f\right), \quad \forall f \in C^{2}(M, \mathbb{R}),
$$

where

$$
\begin{gathered}
\Phi:=\frac{1}{4}\left(\sum_{a=1}^{2} \eta \circ \xi_{a}+\sum_{a=1}^{2} \tilde{\eta} \circ \zeta_{a}\right), \\
\eta(t):=-\ln \left(q_{\kappa}(\widetilde{R})-t\right), \quad \widetilde{\eta}(t):=-\ln \left(1+C_{0}-t\right) . \\
\xi_{a}:=q_{\kappa} \circ \operatorname{dist}\left(y_{0}, \phi_{a}\right), \quad \zeta_{a}:=\left|\psi_{a}\right|^{2}, \quad a=1,2 .
\end{gathered}
$$

Using the definition of $\mathfrak{L}$ and the properties of the functions $\eta$ and $\widetilde{\eta}$, it is easy to verify that (c.f. (2.18) in [18]):

$$
\mathfrak{L}\left(e^{\Phi \widetilde{\xi}}\right) \geq \Delta \widetilde{\xi}+\frac{1}{4} \widetilde{\xi} \sum_{a=1}^{2} \eta^{\prime}\left(\xi_{a}\right) \Delta \xi_{a}+\frac{1}{4} \widetilde{\xi} \sum_{a=1}^{2} \widetilde{\eta}^{\prime}\left(\zeta_{a}\right) \Delta \zeta_{a} .
$$


Our aim is to show that $\mathfrak{L}\left(e^{\Phi \widetilde{\xi}}\right) \geq 0$. We divide the first term on the RHS into two parts: $\Delta \widetilde{\xi}=\Delta \xi+\frac{1}{2} \Delta\left|\psi_{1}-\psi_{2}\right|^{2}$, where

$$
\xi:=q_{v} \circ \operatorname{dist}\left(\phi_{1}, \phi_{2}\right), \quad v:=\frac{\kappa}{4} .
$$

Step 1. Estimate of $\Delta \xi$.

We can build upon the established strategy in the harmonic map case, and the essential point thus is to handle the additional terms on the right hand side of the equations for $\phi_{1}, \phi_{2}$ when compared with ordinary harmonic maps. The resulting error term has to controlled very carefully so that we can later trade it off against other terms.

Set $\widetilde{\phi}:=\phi_{1} \times \phi_{2}$. Then $\rho=\operatorname{dist} \circ \widetilde{\phi}: M \rightarrow \mathbb{R}$. Using (2.19) we have

$$
\begin{aligned}
\Delta \xi & =\Delta(F \circ \widetilde{\phi}) \\
& =\operatorname{trace}_{M} \phi^{*} \nabla^{2} F+\langle\nabla F \circ \widetilde{\phi}, \tau(\widetilde{\phi})\rangle \\
& \geq s_{v}^{\prime}(\rho) \delta^{2}\left(d \phi_{1}, d \phi_{2}\right)-s_{v}(\rho) b_{\kappa}(\rho) \sum_{a=1}^{2}\left|d \phi_{a}\right|^{2}+\langle\nabla F \circ \widetilde{\phi}, \tau(\widetilde{\phi})\rangle .
\end{aligned}
$$

Since

$$
\nabla F \circ \widetilde{\phi}=\left(\nabla\left(q_{v} \circ \text { dist }\right)\right) \circ \widetilde{\phi}=s_{v}(\rho)(\nabla \text { dist }) \circ \widetilde{\phi}
$$

and

$$
\tau(\widetilde{\phi})=\tau\left(\phi_{1}\right) \oplus \tau\left(\phi_{2}\right)=\mathcal{R}_{1} \oplus \mathcal{R}_{2},
$$

here we have used

$$
\mathcal{R}_{a}:=\mathcal{R}\left(\phi_{a}, \psi_{a}\right), \quad a=1,2
$$

to denote the RHS of the $\phi$-equations (1.4), we have

$$
\begin{aligned}
\langle\nabla F \circ \widetilde{\phi}, \tau(\widetilde{\phi})\rangle & \left.=s_{v}(\rho)\langle\nabla \text { dist }) \circ \widetilde{\phi}, \mathcal{R}_{1} \oplus \mathcal{R}_{2}\right\rangle \\
& =s_{\nu}(\rho)\left\langle e_{1}(\widetilde{\phi}) \oplus e_{2}(\widetilde{\phi}), \mathcal{R}_{1} \oplus \mathcal{R}_{2}\right\rangle \\
& \leq s_{v}(\rho) \delta\left(\mathcal{R}_{1}, \mathcal{R}_{2}\right),
\end{aligned}
$$

$e_{1}:=-\sigma^{\prime}(0), e_{2}:=\sigma^{\prime}(\rho)$. In the last step, we have used the following inequality (c.f. (2.13) in [18] ):

$$
\left|\left\langle e_{1} \oplus e_{2}, v_{1} \oplus v_{2}\right\rangle\right|=\left|\left\langle e_{1}, v_{1}\right\rangle+\left\langle e_{2}, v_{2}\right\rangle\right| \leq \delta\left(v_{1}, v_{2}\right) .
$$

Substituting (3.5) into (3.4), we have

$$
\Delta \xi \geq s_{\nu}^{\prime}(\rho) \delta^{2}\left(d \phi_{1}, d \phi_{2}\right)-s_{\nu}(\rho) b_{\kappa}(\rho) \sum_{a=1}^{2}\left|d \phi_{a}\right|^{2}-s_{\nu}(\rho) \delta\left(\mathcal{R}_{1}, \mathcal{R}_{2}\right) .
$$

To estimate the last term in the above inequality, we denote $K_{j k l}^{m}\left(\phi_{a}\right) \equiv R_{j k l}^{i}\left(\phi_{a}\right) a_{i}^{m}\left(\phi_{a}\right)$, $a=1,2$, then $\mathcal{R}_{a}=K_{j k l}^{m}\left(\phi_{a}\right) \phi_{a \alpha}^{j}\left\langle e_{\alpha} \cdot \psi_{a}^{k}, \psi_{a}^{l}\right\rangle E_{m}\left(\phi_{a}\right)$ (recall that the notations $a_{i}^{m}$ and $E_{i}$ were given in the proof of Corollary 2.1), and

$$
\begin{aligned}
\delta_{0}\left(\mathcal{R}_{1}, \mathcal{R}_{2}\right) & \leq \sum_{m}\left|K_{j k l}^{m}\left(\phi_{1}\right) \phi_{1 \alpha}^{j}\left\langle e_{\alpha} \cdot \psi_{1}^{k}, \psi_{1}^{l}\right\rangle-K_{j k l}^{m}\left(\phi_{2}\right) \phi_{2 \alpha}^{j}\left\langle e_{\alpha} \cdot \psi_{2}^{k}, \psi_{2}^{l}\right\rangle\right| \\
& \leq \sum_{m}\left|\left[K_{j k l}^{m}\left(\phi_{1}\right)-K_{j k l}^{m}\left(\phi_{2}\right)\right] \phi_{1 \alpha}^{j}\left\langle e_{\alpha} \cdot \psi_{1}^{k}, \psi_{1}^{l}\right\rangle\right|
\end{aligned}
$$




$$
\begin{aligned}
& \quad+\sum_{m}\left|K_{j k l}^{m}\left(\phi_{2}\right)\left(\phi_{1 \alpha}^{j}-\phi_{2 \alpha}^{j}\right)\left\langle e_{\alpha} \cdot \psi_{1}^{k}, \psi_{1}^{l}\right\rangle\right| \\
& +\sum_{m}\left|K_{j k l}^{m}\left(\phi_{2}\right) \phi_{2 \alpha}^{j}\left\langle e_{\alpha} \cdot\left(\psi_{1}^{k}-\psi_{2}^{k}\right), \psi_{1}^{l}\right\rangle\right| \\
& \quad+\sum_{m}\left|K_{j k l}^{m}\left(\phi_{2}\right) \phi_{2 \alpha}^{j}\left\langle e_{\alpha} \cdot \psi_{2}^{k}, \psi_{1}^{l}-\psi_{2}^{l}\right\rangle\right| \\
& \leq C \rho\left|d \phi_{1}\right|\left|\psi_{1}\right|^{2}+C\left|d \phi_{1}-d \phi_{2}\right|\left|\psi_{1}\right|^{2}+C\left|d \phi_{2}\right|\left(\left|\psi_{1}\right|+\left|\psi_{2}\right|\right)\left|\psi_{1}-\psi_{2}\right|,
\end{aligned}
$$

by Lemma 2.1, and utilizing the Young's inequality, we have

$$
\begin{aligned}
\delta\left(\mathcal{R}_{1}, \mathcal{R}_{2}\right) & \leq \delta_{0}\left(\mathcal{R}_{1}, \mathcal{R}_{2}\right)+C \rho\left(\left|\mathcal{R}_{1}\right|+\left|\mathcal{R}_{2}\right|\right) \\
& \leq C \rho \sum_{a}\left(\left|d \phi_{a}\right|^{2}+\left|\psi_{a}\right|^{4}\right)+C\left|d \phi_{1}-d \phi_{2}\right|\left|\psi_{1}\right|^{2}+C_{S}\left|d \phi_{2}\right|\left|\psi_{1}-\psi_{2}\right|
\end{aligned}
$$

here and in the sequel, we use $C$ and $C_{S}$ to denote positive constants depending only on $n, n^{\prime}, y_{0}, R, C_{0}, M, N$. In some cases (such as (3.33) bellow), they may also depend on an integer $p>0 . C_{S}$ is small when $R$ and $C_{0}$ are small. $C, C_{S}$ may change values from line to line. For our purpose, we will not concern the concrete values of them. From (3.8) and the fact that $s_{\nu}(\rho) \leq \rho$, we have

$$
\begin{aligned}
s_{v}(\rho) \delta\left(\mathcal{R}_{1}, \mathcal{R}_{2}\right) \leq & C \rho^{2} \sum_{a}\left(\left|d \phi_{a}\right|^{2}+\left|\psi_{a}\right|^{4}\right)+\frac{C}{\varepsilon_{1}} \rho^{2} \sum_{a}\left|\psi_{a}\right|^{4}+C \varepsilon_{1}\left|d \phi_{1}-d \phi_{2}\right|^{2} \\
& +C_{S} \rho^{2} \sum_{a}\left|d \phi_{a}\right|^{2}+C_{S}\left|\psi_{1}-\psi_{2}\right|^{2}
\end{aligned}
$$

where $\varepsilon_{1}>0$ is a small constant to be chosen later.

Now we turn to the second term of the RHS of (3.6). Using (2.20), (2.21), and the elementary identities (recall: $v:=\frac{\kappa}{4}$ )

$$
a_{\kappa}(t)=\frac{\kappa}{2} \cdot \frac{s_{v}(t)}{s_{v}^{\prime}(t)}, \quad \frac{s_{v}^{2}}{2 q_{v} s_{v}^{\prime}}=\frac{1}{2}\left(1+\frac{1}{s_{v}^{\prime}}\right),
$$

one can easily obtain

$$
\frac{s_{\nu}(\rho) b_{\kappa}(\rho)}{q_{\kappa}(\rho)}=\frac{\kappa}{2}\left(1+\frac{1}{s_{v}^{\prime}(\rho)}\right)\left(1+\frac{\rho}{s_{\kappa}(\rho)}\right)
$$

and consequently

$$
s_{\nu}(\rho) b_{\kappa}(\rho) \leq C \rho^{2} .
$$

Substituting (3.9), (3.12) into (3.6), and using (2.14), we obtain

$$
\Delta \xi \geq\left(s_{\kappa}^{\prime}(\rho)-C \varepsilon_{1}\right) \delta^{2}\left(d \phi_{1}, d \phi_{2}\right)-\frac{C}{\varepsilon_{1}} \rho^{2} \sum_{a}\left(\left|d \phi_{a}\right|^{2}+\left|\psi_{a}\right|^{4}\right)-C_{S}\left|\psi_{1}-\psi_{2}\right|^{2} .
$$


Step 2. Estimate of $\Delta \frac{1}{2}\left|\psi_{1}-\psi_{2}\right|^{2}$.

By the Lichnerowicz formula (see e.g. [21]), we have

$$
\begin{aligned}
\frac{1}{2} \Delta\left|\psi_{1}-\psi_{2}\right|^{2} & \equiv \frac{1}{2} \sum_{i} \Delta\left|\psi_{1}^{i}-\psi_{2}^{i}\right|^{2} \\
& =\sum_{i}\left|\nabla\left(\psi_{1}^{i}-\psi_{2}^{i}\right)\right|^{2}+\sum_{i}\left\langle\nabla^{2}\left(\psi_{1}^{i}-\psi_{2}^{i}\right), \psi_{1}^{i}-\psi_{2}^{i}\right\rangle \\
& =\sum_{i}\left|\nabla\left(\psi_{1}^{i}-\psi_{2}^{i}\right)\right|^{2}+\sum_{i} \frac{S_{M}}{4}\left|\psi_{1}^{i}-\psi_{2}^{i}\right|^{2}-\sum_{i}\left\langle\partial^{2}\left(\psi_{1}^{i}-\psi_{2}^{i}\right), \psi_{1}^{i}-\psi_{2}^{i}\right\rangle \\
& \equiv\left|\nabla\left(\psi_{1}-\psi_{2}\right)\right|^{2}+\frac{S_{M}}{4}\left|\psi_{1}-\psi_{2}\right|^{2}-\sum_{i}\left\langle\partial^{2}\left(\psi_{1}^{i}-\psi_{2}^{i}\right), \psi_{1}^{i}-\psi_{2}^{i}\right\rangle .
\end{aligned}
$$

The first two terms are good, because positive. (For the positivity of the second term, we need our scalar curvature assumption.) The last term still needs to be controlled. In fact, this term mixes the $\phi$ and the $\psi$ fields because the Dirac operator depends on $\phi$. To deal with this term, we shall need estimates for $|\nabla \phi|$ and $|\nabla \psi|$ for a Dirac-harmonic map $(\phi, \psi)$ satisfying (1.6) as stated in the following lemma.

Lemma 3.1 Let $(\phi, \psi)$ be as in Theorem 1.1. Then

$$
\max _{M}|\nabla \phi|, \quad \max _{M}|\nabla \psi| \leq C\left(n, n^{\prime}, y_{0}, R, C_{0}, M, N\right)
$$

for some positive constant $C\left(n, n^{\prime}, y_{0}, R, C_{0}, M, N\right)$.

We leave the proof to the Appendix and continue to prove Theorem 1.1. From the $\psi$-equation $\not \partial \psi_{1}^{i}=-\Gamma_{j k}^{i}\left(\phi_{1}\right) \nabla \phi_{1}^{j} \cdot \psi_{1}^{k}$, we have

$$
\begin{aligned}
\partial^{2} \psi_{1}^{i}= & -\Gamma_{j k, l}^{i}\left(\phi_{1}\right) \nabla \phi_{1}^{l} \cdot \nabla \phi_{1}^{j} \cdot \psi_{1}^{k}-\Gamma_{j k}^{i}\left(\phi_{1}\right) e_{\alpha} \cdot \nabla_{e_{\alpha}}\left(\nabla \phi_{1}^{j}\right) \cdot \psi_{1}^{k} \\
& -\Gamma_{j k}^{i}\left(\phi_{1}\right) e_{\alpha} \cdot \nabla \phi_{1}^{j} \cdot \psi_{1 \alpha}^{k} .
\end{aligned}
$$

Noting that

$$
\begin{aligned}
e_{\alpha} \cdot \nabla_{e_{\alpha}}\left(\nabla \phi_{1}^{j}\right) \cdot \psi_{1}^{k} & =e_{\alpha} \cdot\left\langle\nabla_{e_{\alpha}}\left(\nabla \phi_{1}^{j}\right), e_{\beta}\right\rangle \cdot \psi_{1}^{k} \\
& =\operatorname{Hess}\left(\phi_{1}^{j}\right)\left(e_{\alpha}, e_{\beta}\right) e_{\alpha} \cdot e_{\beta} \cdot \psi_{1}^{k} \\
& =-\Delta \phi_{1}^{j} \psi_{1}^{k},
\end{aligned}
$$

we have

$$
\begin{aligned}
\not p^{2} \psi_{1}^{i}= & -\Gamma_{j k, l}^{i}\left(\phi_{1}\right) \nabla \phi_{1}^{l} \cdot \nabla \phi_{1}^{j} \cdot \psi_{1}^{k}+\Gamma_{j k}^{i}\left(\phi_{1}\right) \Delta \phi_{1}^{j} \psi_{1}^{k} \\
& -\Gamma_{j k}^{i}\left(\phi_{1}\right) e_{\alpha} \cdot \nabla \phi_{1}^{j} \cdot \psi_{1 \alpha}^{k} .
\end{aligned}
$$

Similarly,

$$
\begin{aligned}
p^{2} \psi_{2}^{i}= & -\Gamma_{j k, l}^{i}\left(\phi_{2}\right) \nabla \phi_{2}^{l} \cdot \nabla \phi_{2}^{j} \cdot \psi_{2}^{k}+\Gamma_{j k}^{i}\left(\phi_{2}\right) \Delta \phi_{2}^{j} \psi_{2}^{k} \\
& -\Gamma_{j k}^{i}\left(\phi_{2}\right) e_{\alpha} \cdot \nabla \phi_{2}^{j} \cdot \psi_{2 \alpha}^{k} .
\end{aligned}
$$


Hence

$$
\begin{gathered}
\left\langle\partial^{2}\left(\psi_{1}^{i}-\psi_{2}^{i}\right), \psi_{1}^{i}-\psi_{2}^{i}\right\rangle=\left\langle\Gamma_{j k, l}^{i}\left(\phi_{2}\right) \nabla \phi_{2}^{l} \cdot \nabla \phi_{2}^{j} \cdot \psi_{2}^{k}-\Gamma_{j k, l}^{i}\left(\phi_{1}\right) \nabla \phi_{1}^{l} \cdot \nabla \phi_{1}^{j} \cdot \psi_{1}^{k}, \psi_{1}^{i}-\psi_{2}^{i}\right\rangle \\
\quad-\left\langle\Gamma_{j k}^{i}\left(\phi_{2}\right) \Delta \phi_{2}^{j} \psi_{2}^{k}-\Gamma_{j k}^{i}\left(\phi_{1}\right) \Delta \phi_{1}^{j} \psi_{1}^{k}, \psi_{1}^{i}-\psi_{2}^{i}\right\rangle \\
\quad+\left\langle\Gamma_{j k}^{i}\left(\phi_{2}\right) e_{\alpha} \cdot \nabla \phi_{2}^{j} \cdot \psi_{2 \alpha}^{k}-\Gamma_{j k}^{i}\left(\phi_{1}\right) e_{\alpha} \cdot \nabla \phi_{1}^{j} \cdot \psi_{1 \alpha}^{k}, \psi_{1}^{i}-\psi_{2}^{i}\right\rangle \\
:=I+J+K . \\
I=\left\langle\left(\Gamma_{j k, l}^{i}\left(\phi_{2}\right)-\Gamma_{j k, l}^{i}\left(\phi_{1}\right)\right) \nabla \phi_{2}^{l} \cdot \nabla \phi_{2}^{j} \cdot \psi_{2}^{k}, \psi_{1}^{i}-\psi_{2}^{i}\right\rangle \\
+\left\langle\Gamma_{j k, l}^{i}\left(\phi_{1}\right)\left(\nabla \phi_{2}^{l}-\nabla \phi_{1}^{l}\right) \cdot \nabla \phi_{2}^{j} \cdot \psi_{2}^{k}, \psi_{1}^{i}-\psi_{2}^{i}\right\rangle \\
+\left\langle\Gamma_{j k, l}^{i}\left(\phi_{1}\right) \nabla \phi_{1}^{l} \cdot\left(\nabla \phi_{2}^{j}-\nabla \phi_{1}^{j}\right) \cdot \psi_{2}^{k}, \psi_{1}^{i}-\psi_{2}^{i}\right\rangle \\
+\left\langle\Gamma_{j k, l}^{i}\left(\phi_{1}\right) \nabla \phi_{2}^{l} \cdot \nabla \phi_{2}^{j} \cdot\left(\psi_{2}^{k}-\psi_{1}^{k}\right), \psi_{1}^{i}-\psi_{2}^{i}\right\rangle \\
:=
\end{gathered}
$$

When $C_{0}$ is small, we have

$$
\begin{aligned}
\left|I_{1}\right| & \leq C \rho\left|d \phi_{2}\right|^{2}\left|\psi_{2}\right|\left|\psi_{1}-\psi_{2}\right| \leq C_{S} \tilde{\xi} \sum_{a}\left|d \phi_{a}\right|^{2}, \\
\left|I_{2}\right| & \leq C\left|d \phi_{1}-d \phi_{2}\right|\left|d \phi_{2}\right|\left|\psi_{2}\right|\left|\psi_{1}-\psi_{2}\right| \\
& =C_{S}\left|d \phi_{1}-d \phi_{2}\right|^{2}+C_{S}\left|\psi_{1}-\psi_{2}\right|^{2} \sum_{a}\left|d \phi_{a}\right|^{2} \\
& \leq C_{S} \delta^{2}\left(d \phi_{1}, d \phi_{2}\right)+C_{S} \widetilde{\xi} \sum_{a}\left|d \phi_{a}\right|^{2} \quad(\text { by (2.14)). }
\end{aligned}
$$

Similarly,

$$
\begin{aligned}
& \left|I_{3}\right| \leq C_{S} \delta^{2}\left(d \phi_{1}, d \phi_{2}\right)+C_{S} \widetilde{\xi} \sum_{a}\left|d \phi_{a}\right|^{2} . \\
& \left|I_{4}\right| \leq C\left|d \phi_{1}\right|^{2}\left|\psi_{1}-\psi_{2}\right|^{2} \leq C \sum_{a}\left|d \phi_{a}\right|^{2}\left|\psi_{1}-\psi_{2}\right|^{2} .
\end{aligned}
$$

Summing up the above four inequalities, we obtain

$$
|I| \leq C_{S} \delta^{2}\left(d \phi_{1}, d \phi_{2}\right)+C \widetilde{\xi} \sum_{a}\left|d \phi_{a}\right|^{2}
$$

By similar arguments (see Appendix for details), we have

$$
\begin{aligned}
& |J| \leq C_{S} \widetilde{\xi} \sum_{a}\left(\left|d \phi_{a}\right|^{2}+\left|\psi_{a}\right|^{4}\right)+C_{S} \delta^{2}\left(d \phi_{1}, d \phi_{2}\right)+C_{S}\left|\psi_{1}-\psi_{2}\right|^{2}, \\
|K| \leq & C \rho\left|d \phi_{2}\right|\left|\nabla \psi_{2}\right|\left|\psi_{1}-\psi_{2}\right|+C_{S}\left|d \phi_{1}-d \phi_{2}\right|\left|\nabla \psi_{2}\right|\left|\psi_{1}-\psi_{2}\right| \\
& +C_{S}\left|d \phi_{1}\right|\left|\nabla\left(\psi_{1}-\psi_{2}\right)\right|\left|\psi_{1}-\psi_{2}\right| \\
\leq & C_{S} \delta^{2}\left(d \phi_{1}, d \phi_{2}\right)+\frac{C}{\varepsilon} \xi \sum_{a}\left|d \phi_{a}\right|^{2}+C_{S}\left|\nabla\left(\psi_{1}-\psi_{2}\right)\right|^{2}+\left(C \varepsilon+C_{S}\right)\left|\psi_{1}-\psi_{2}\right|^{2},
\end{aligned}
$$

where $\varepsilon>0$ is a small constant to be chosen later, and in the last step, we have used (3.15). 
Substituting (3.19), (3.20) and (3.21) into (3.14), we obtain

$$
\begin{aligned}
\frac{1}{2} \Delta\left|\psi_{1}-\psi_{2}\right|^{2} \geq & \left|\nabla\left(\psi_{1}-\psi_{2}\right)\right|^{2}+\frac{S_{M}}{4}\left|\psi_{1}-\psi_{2}\right|^{2}-(|I|+|J|+|K|) \\
\geq & \left|\nabla\left(\psi_{1}-\psi_{2}\right)\right|^{2}+\frac{S_{M}}{4}\left|\psi_{1}-\psi_{2}\right|^{2}-C_{S} \delta^{2}\left(d \phi_{1}, d \phi_{2}\right)-\frac{C}{\varepsilon} \widetilde{\xi} \sum_{a}\left|d \phi_{a}\right|^{2} \\
& -C_{S} \widetilde{\xi} \sum_{a}\left|\psi_{a}\right|^{4}-\left(C_{S}+C \varepsilon\right)\left|\psi_{1}-\psi_{2}\right|^{2}-C_{S}\left|\nabla\left(\psi_{1}-\psi_{2}\right)\right|^{2} \\
\geq & \left(\frac{1}{4} S_{M}-C_{S}-C \varepsilon\right)\left|\psi_{1}-\psi_{2}\right|^{2}-\frac{C}{\varepsilon} \widetilde{\xi} \sum_{a}\left(\left|d \phi_{a}\right|^{2}+\left|\psi_{a}\right|^{4}\right) \\
& -C_{S} \delta^{2}\left(d \phi_{1}, d \phi_{2}\right) .
\end{aligned}
$$

Combining (3.13) and (3.22), we conclude

$$
\begin{aligned}
\Delta \widetilde{\xi} \geq & \left(\frac{1}{4} \inf S_{M}-C_{S}-C \varepsilon\right)\left|\psi_{1}-\psi_{2}\right|^{2}+\left(s_{\kappa}^{\prime}(\rho)-C \varepsilon_{1}-C_{S}\right) \delta^{2}\left(d \phi_{1}, d \phi_{2}\right) \\
& -C\left(\frac{1}{\varepsilon}+\frac{1}{\varepsilon_{1}}\right) \widetilde{\xi} \sum_{a}\left(\left|d \phi_{a}\right|^{2}+\left|\psi_{a}\right|^{4}\right)
\end{aligned}
$$

choosing $\varepsilon, \varepsilon_{1}, R$ and $C_{0}$ small enough, we then have

$$
\Delta \widetilde{\xi} \geq-C \widetilde{\xi} \sum_{a}\left(\left|d \phi_{a}\right|^{2}+\left|\psi_{a}\right|^{4}\right)
$$

Step 3. Estimates of $\Delta \xi_{a}$ and $\Delta \zeta_{a}$

These terms are somewhat easier because they only involve one of the Dirac-harmonic pairs. For the $\phi$-fields, this can also be seen as controlling the distance from a constant field (compare (2.18) with (2.19) and note that the former does not need the pseudo distance $\delta$ ).

Back to (3.3). For the second term in the RHS of (3.3), by Lemma 2.2, we have

$$
\begin{aligned}
\Delta \xi_{a} & =\Delta\left(G \circ \phi_{a}\right) \\
& =\operatorname{trace}_{M} \phi_{a}^{*} \nabla^{2} G+\left\langle(\nabla G)\left(\phi_{a}\right), \tau\left(\phi_{a}\right)\right\rangle \\
& \geq s_{\kappa}^{\prime}\left(\rho_{a}\right)\left|d \phi_{a}\right|^{2}+\left\langle(\nabla G)\left(\phi_{a}\right), \tau\left(\phi_{a}\right)\right\rangle .
\end{aligned}
$$

Using $(\nabla G)\left(\phi_{a}\right)=s_{\kappa}\left(\rho_{a}\right)\left(\nabla \operatorname{dist}\left(y_{0}, \cdot\right)\right)\left(\phi_{a}\right)$, we conclude

$$
\Delta \xi_{a} \geq s_{\kappa}^{\prime}\left(\rho_{a}\right)\left|d \phi_{a}\right|^{2}-C s_{\kappa}\left(\rho_{a}\right)\left|d \phi_{a} \| \psi_{a}\right|^{2}, \quad a=1,2 .
$$

As for the third term of the RHS of (3.3), using the Weitzenböck formula in [8], we have

$$
\begin{aligned}
\Delta\left|\psi_{a}\right|^{2} & =\left|\widetilde{\nabla} \psi_{a}\right|^{2}+\frac{S_{M}}{4}\left|\psi_{a}\right|^{2}-\frac{1}{2} R_{m i j k}\left\langle\nabla \phi_{a}^{k} \cdot \psi_{a}^{m}, \nabla \phi_{a}^{l} \cdot \psi_{a}^{j}\right\rangle \\
& \geq \frac{S_{M}}{4}\left|\psi_{a}\right|^{2}-C\left|d \phi_{a}\right|^{2}\left|\psi_{a}\right|^{2}, \quad a=1,2,
\end{aligned}
$$

where $C>0$ is a constant.

We can now combine our estimates and obtain the desired inequality. Substituting (3.24), (3.26) and (3.27) into (3.3) and noting that $s_{\kappa}^{\prime}\left(\rho_{a}\right)=1-\kappa q_{\kappa}\left(\rho_{a}\right), a=1,2$, 
we deduce that

$$
\begin{aligned}
\mathfrak{L}\left(e^{\Phi} \widetilde{\xi}\right) \geq & \frac{\widetilde{\xi}}{4} \sum_{a}\left[\frac{1-\kappa q_{\kappa}\left(\rho_{a}\right)}{q_{\kappa}(\widetilde{R})-q_{\kappa}\left(\rho_{a}\right)}-4 C-\frac{C\left|\psi_{a}\right|^{2}}{1+C_{0}-\left|\psi_{a}\right|^{2}}\right]\left|d \phi_{a}\right|^{2} \\
& +\frac{\widetilde{\xi}}{4} \sum_{a}\left[\frac{\inf S_{M}}{4\left(1+C_{0}-\left|\psi_{a}\right|^{2}\right)}-\frac{C s_{\kappa}\left(\rho_{a}\right)\left|\psi_{a}\right|^{2}}{q_{\kappa}(\widetilde{R})-q_{\kappa}\left(\rho_{a}\right)}-4 C\left|\psi_{a}\right|^{2}\right]\left|\psi_{a}\right|^{2} .
\end{aligned}
$$

It is not hard to see that by taking $R$ small (so that $\widetilde{R} \in(R, \pi / 2 \sqrt{\kappa})$ is also small), and then choosing $C_{0}$ sufficiently small, we can conclude that

$$
\mathfrak{L}(\Theta) \equiv \mathfrak{L}\left(e^{\Phi \widetilde{\xi}}\right) \geq 0
$$

In particular, we see that we need to assume that $\left|\psi_{a}\right|$ be sufficiently small so that we can absorb the last term in the first sum by the convexity properties of the target and the last term in the second sum by the positive scalar curvature of the domain.

Therefore, by Hopf's maximum principle, the function $\Theta$ satisfies

$$
\sup _{M} \Theta \leq \sup _{\partial M} \Theta .
$$

Having thus achieved (1.13), we can now prove the global estimate (1.14). This will follow from (1.13), essentially by utilizing the equations and applying the Sobolev embedding theorem and the elliptic estimate (2.27).

From the above inequality (3.30), we deduce the following estimate:

$$
\left\|\phi_{1}-\phi_{2}\right\|_{C^{0}(M)}+\left\|\psi_{1}-\psi_{2}\right\|_{C^{0}(M)} \leq C\left(\left\|\phi_{1}-\phi_{2}\right\|_{C^{0}(\partial M)}+\left\|\psi_{1}-\psi_{2}\right\|_{C^{0}(\partial M)}\right)
$$

for some constant $C>0$.

Note that from

$$
\begin{aligned}
\not \partial\left(\psi_{1}^{i}-\psi_{2}^{i}\right)= & \Gamma_{j k}^{i}\left(\phi_{2}\right) \nabla \phi_{2}^{j} \cdot \psi_{2}^{k}-\Gamma_{j k}^{i}\left(\phi_{1}\right) \nabla \phi_{1}^{j} \cdot \psi_{1}^{k} \\
= & \left(\Gamma_{j k}^{i}\left(\phi_{2}\right)-\Gamma_{j k}^{i}\left(\phi_{1}\right)\right) \nabla \phi_{2}^{j} \cdot \psi_{2}^{k}+\Gamma_{j k}^{i}\left(\phi_{1}\right)\left(\nabla \phi_{2}^{j}-\nabla \phi_{1}^{j}\right) \cdot \psi_{2}^{k} \\
& +\Gamma_{j k}^{i}\left(\phi_{1}\right) \nabla \phi_{1}^{j} \cdot\left(\psi_{2}^{k}-\psi_{1}^{k}\right)
\end{aligned}
$$

and (3.15), it follows that

$$
\begin{aligned}
\left\|\not \partial\left(\psi_{1}^{i}-\psi_{2}^{i}\right)\right\|_{C^{0}(M)} \leq & C_{S}\left(\left\|\phi_{1}-\phi_{2}\right\|_{C^{0}(M)}+\left\|d \phi_{1}-d \phi_{2}\right\|_{C^{0}(M)}\right. \\
& \left.+\left\|\psi_{1}-\psi_{2}\right\|_{C^{0}(M)}\right), \quad i=1,2, \cdots, n^{\prime} .
\end{aligned}
$$

From (2.27), for any $p>n$, we have

$$
\left\|\psi_{1}^{i}-\psi_{2}^{i}\right\|_{H^{1, p}(M)} \leq C\left(\left\|\not \partial\left(\psi_{1}^{i}-\psi_{2}^{i}\right)\right\|_{L^{p}(M)}+\left\|\mathbf{B}\left(\psi_{1}^{i}-\psi_{2}^{i}\right)\right\|_{H^{1-\frac{1}{p}, p}(\partial M)}\right) .
$$

By the Sobolev embedding theorem and (3.32), we obtain

$$
\begin{aligned}
\left\|\psi_{1}-\psi_{2}\right\|_{C^{0}(M)} \leq & C_{S}\left\|\phi_{1}-\phi_{2}\right\|_{C^{0}(M)}+C_{S}\left\|d \phi_{1}-d \phi_{2}\right\|_{C^{0}(M)} \\
& +C\left\|\mathbf{B}\left(\psi_{1}-\psi_{2}\right)\right\|_{H^{1-\frac{1}{p}, p}(\partial M)}
\end{aligned}
$$


From this and (3.31), we have

$$
\begin{aligned}
\left\|\phi_{1}-\phi_{2}\right\|_{C^{0}(M)}+\left\|\psi_{1}-\psi_{2}\right\|_{C^{0}(M)} \leq & C\left\|\phi_{1}-\phi_{2}\right\|_{C^{0}(\partial M)}+C\left\|\mathbf{B}\left(\psi_{1}-\psi_{2}\right)\right\|_{H^{1-\frac{1}{p}, p}(\partial M)} \\
& +C_{S}\left\|d \phi_{1}-d \phi_{2}\right\|_{C^{0}(M)} .
\end{aligned}
$$

On the other hand,

$$
\begin{aligned}
\Delta\left(\phi_{1}^{i}-\phi_{2}^{i}\right)= & \Gamma_{j k}^{i}\left(\phi_{2}\right) \phi_{2 \alpha}^{j} \phi_{2 \beta}^{k} g^{\alpha \beta}-\Gamma_{j k}^{i}\left(\phi_{1}\right) \phi_{1 \alpha}^{j} \phi_{1 \beta}^{k} g^{\alpha \beta} \\
& +\frac{1}{2} R_{j k l}^{i}\left(\phi_{1}\right)\left\langle\nabla \phi_{1}^{j} \cdot \psi_{1}^{k}, \psi_{1}^{l}\right\rangle-\frac{1}{2} R_{j k l}^{i}\left(\phi_{2}\right)\left\langle\nabla \phi_{2}^{j} \cdot \psi_{1}^{k}, \psi_{2}^{l}\right\rangle,
\end{aligned}
$$

using (3.15) and the smallness of $R$ and $C_{0}$, it follows that

$$
\left|\Delta\left(\phi_{1}-\phi_{2}\right)\right| \leq C\left|\phi_{1}-\phi_{2}\right|+C_{S}\left|d \phi_{1}-d \phi_{2}\right|+C_{S}\left|\psi_{1}-\psi_{2}\right| .
$$

Hence, for any $p>n$,

$$
\begin{aligned}
\left\|\phi_{1}-\phi_{2}\right\|_{H^{2, p}(M)} \leq & C\left\|\phi_{1}-\phi_{2}\right\|_{L^{p}(M)}+C_{S}\left\|d \phi_{1}-d \phi_{2}\right\|_{L^{p}(M)} \\
& +C_{S}\left\|\psi_{1}-\psi_{2}\right\|_{L^{p}(M)}+C\left\|\phi_{1}-\phi_{2}\right\|_{H^{2-\frac{1}{p}, p}(\partial M)}
\end{aligned} .
$$

By Sobolev embedding, we conclude that

$$
\begin{aligned}
\left\|d \phi_{1}-d \phi_{2}\right\|_{C^{0}(M)} \leq & C\left\|\phi_{1}-\phi_{2}\right\|_{C^{0}(M)}+C_{S}\left\|\psi_{1}-\psi_{2}\right\|_{C^{0}(M)} \\
& +C\left\|\phi_{1}-\phi_{2}\right\|_{H^{2-\frac{1}{p}, p}(\partial M)}
\end{aligned}
$$

Substituting this into (3.34) and using the Sobolev embedding again, we obtain

$$
\begin{aligned}
& \left\|\phi_{1}-\phi_{2}\right\|_{C^{0}(M)}+\left\|\psi_{1}-\psi_{2}\right\|_{C^{0}(M)} \\
& \quad \leq C\left(\left\|\phi_{1}-\phi_{2}\right\|_{H^{2-\frac{1}{p}, p}(\partial M)}+\left\|\mathbf{B} \psi_{1}-\mathbf{B} \psi_{2}\right\|_{H^{1-\frac{1}{p}, p}(\partial M)}\right)
\end{aligned}
$$

for some constant $C=C\left(n, n^{\prime}, p, y_{0}, R, C_{0}, M, N\right)>0$. The uniqueness is a direct consequence of the above inequality. This completes the proof of Theorem 1.1.

\section{Existence for boundary value problems of Dirac-harmonic maps}

In this section, we will prove existence theorem for Dirac-harmonic maps satisfying the boundary condition (1.17) in Theorem 1.2, namely:

$$
\left\{\begin{array}{l}
\left.\phi\right|_{\partial M}=\left.\phi_{0}\right|_{\partial M} \\
\mathbf{B} \psi=\mathbf{B} \psi_{0}
\end{array}\right.
$$

for any given $\phi_{0} \in H^{2, p}(M, N)$ with $\phi_{0}(M) \subset B_{R}\left(y_{0}\right)$ and $\psi_{0} \in H^{1, p}\left(\Sigma M \otimes \phi_{0}^{-1}(T N)\right)$ with $\left|\psi_{0}\right|^{2}<C_{0}, p>n:=\operatorname{dim} M$.

As already explained in the introduction, we need to employ a continuity method, because we neither have a variational method nor a heat equation technique at our disposal. Essentially, we shall multiply the nonlinear terms in our equations by a factor $\tau$ which we shall let increase from 0 to 1 . Of course, we shall have to be careful with the boundary conditions as well as with the geometric interpretations in terms of convexity conditions. 
Choose the normal coordinates $\left\{y^{i}\right\}_{i=1,2, \cdots, n^{\prime}}$ centered at $y_{0}$, we can then write

$$
\begin{aligned}
& \phi_{0}:=\left(\phi_{0}^{1}, \phi_{0}^{2}, \cdots, \phi_{0}^{n^{\prime}}\right) \in\left(H^{2, p}(M)\right)^{n^{\prime}} \equiv \overbrace{H^{2, p}(M) \times \cdots \times H^{2, p}(M)}^{n^{\prime}}, \\
& \psi_{0}:=\left(\psi_{0}^{1}, \psi_{0}^{2}, \cdots, \psi_{0}^{n^{\prime}}\right) \in\left(H^{1, p}(\Sigma M)\right)^{n^{\prime}} \equiv \overbrace{H^{1, p}(\Sigma M) \times \cdots \times H^{1, p}(\Sigma M)}^{n^{\prime}} .
\end{aligned}
$$

For any Dirac-harmonic map $(\phi, \psi) \in H^{2, p}(M, N) \times H^{1, p}\left(\Sigma M \otimes \phi^{-1}(T N)\right)$ with $\phi(M) \subset$ $B_{R}\left(y_{0}\right)$ we can also write

$$
\phi:=\left(\phi^{1}, \cdots, \phi^{n^{\prime}}\right) \in\left(H^{2, p}(M)\right)^{n^{\prime}}, \quad \psi:=\left(\psi^{1}, \cdots, \psi^{n^{\prime}}\right) \in\left(H^{1, p}(\Sigma M)\right)^{n^{\prime}} .
$$

In these notations, $\phi$ and $\psi$ can be viewed as vectors in $\mathbb{R}^{n^{\prime}}$ and $\Sigma M \otimes \mathbb{R}^{n^{\prime}}$ respectively, and we have:

$$
E_{1}\left|\phi_{1}-\phi_{2}\right| \leq \operatorname{dist}\left(\phi_{1}, \phi_{2}\right) \leq E_{2}\left|\phi_{1}-\phi_{2}\right|, \quad E_{1}|\psi| \leq|\psi|_{\Sigma M \otimes \phi^{-1}(T N)} \leq E_{2}|\psi|,
$$

where $E_{1}$ and $E_{2}$ are positive constants depending only on $N, y_{0}$, and $\kappa$, and $|\cdot|$ denotes the standard norms in $\mathbb{R}^{n^{\prime}}$ or $\Sigma M \otimes \mathbb{R}^{n^{\prime}}$.

Proof of Theorem 1.2. We first note that by the same proof as that of Theorem 1.1, one can find positive constants $R<\pi / 2 \sqrt{\kappa}$ and $C_{0} \leq 1$ such that for all $\tau \in[0,1]$, the solutions of

$$
\left\{\begin{array}{l}
\Delta \phi^{i}+\tau \Gamma_{j k}^{i} \phi_{\alpha}^{j} \phi_{\beta}^{k} g^{\alpha \beta}+\frac{\tau}{2} R_{j k l}^{i}\left\langle\psi^{k}, \nabla \phi^{j} \cdot \psi^{l}\right\rangle=0 \\
\not \partial \psi^{i}+\tau \Gamma_{j k}^{i} \nabla \phi^{j} \cdot \psi^{k}=0, \quad i=1,2, \cdots, n^{\prime}
\end{array}\right.
$$

with the corresponding smallness conditions on $\phi$ and $\psi\left(\phi(M) \subset B_{R}\left(y_{0}\right)\right.$ and $\left.|\psi|^{2}<C_{0}\right)$ also satisfy the maximum principle in Theorem 1.1. We choose $\left\|\phi_{0}\right\|_{H^{2, p}(M)}$ and $\left\|\psi_{0}\right\|_{H^{1, p}(M)}$ small such that $\phi_{0}(M) \subset B_{R}\left(y_{0}\right)$ and $\left|\psi_{0}\right|^{2}<C_{0}$.

As in [31], for any two real parameters $\lambda, \tau$ with $(\lambda, \tau) \in[0,1] \times[0,1]$, we consider the following boundary value problems

$$
P_{R, C_{0}}^{\tau, \lambda}\left(\phi_{0}, \psi_{0}\right):\left\{\begin{array}{l}
\left\{\begin{array}{l}
\Delta \phi^{i}+\tau \Gamma_{j k}^{i} \phi_{\alpha}^{j} \phi_{\beta}^{k} g^{\alpha \beta}+\frac{\tau}{2} R_{j k l}^{i}\left\langle\psi^{k}, \nabla \phi^{j} \cdot \psi^{l}\right\rangle=0, \\
\not \partial \psi^{i}+\tau \Gamma_{j k}^{i} \nabla \phi^{j} \cdot \psi^{k}=0, \quad i=1,2, \cdots, n^{\prime} ;
\end{array}\right. \\
\left\{\begin{array}{l}
\phi-\tau \lambda \phi_{0} \in\left(H_{0}^{2, p}(M)\right)^{n^{\prime}}, \\
\mathbf{B}\left(\psi-\tau \lambda \psi_{0}\right)=0 ; \\
\left\{\begin{array}{l}
\phi(M) \subset B_{R}\left(y_{0}\right), \\
|\psi|^{2}<C_{0} .
\end{array}\right.
\end{array}\right.
\end{array}\right.
$$

Denote by $\Lambda$ the set of all $\lambda \in[0,1]$ for which the problem $P_{R, C_{0}}^{\tau, \lambda}\left(\phi_{0}, \psi_{0}\right)$ has a solution $(\phi, \psi) \in\left(H^{2, p}(M)\right)^{n^{\prime}} \times\left(H^{1, p}(\Sigma M)\right)^{n^{\prime}}$.

Let $\Lambda^{*}$ be the set of all $\lambda \in \Lambda$ such that

$$
\left\{\begin{array}{l}
{[0, \lambda] \subset \Lambda,} \\
\left\|\phi_{\tilde{\lambda}}\right\|_{2, p}+\left\|\psi_{\tilde{\lambda}}\right\|_{1, p}<C\left(n, n^{\prime}, p, \phi_{0}, \psi_{0}, y_{0}, R, C_{0}, M, N, \lambda\right), \quad 0 \leq \tilde{\lambda} \leq \lambda,
\end{array}\right.
$$


where $\left(\phi_{\tilde{\lambda}}, \psi_{\tilde{\lambda}}\right)$ denotes the solutions of $P_{R, C_{0}}^{\tau, \tilde{\lambda}}\left(\phi_{0}, \psi_{0}\right)$, and $C(\cdots)$ is a positive constant depending only on the corresponding quantities in the bracket.

Our aim is to show that $\Lambda^{*}=[0,1]$, from which the conclusion of Theorem 1.2 follows.

Clearly $\Lambda^{*}$ is not empty, since $0 \in \Lambda^{*}$. Suppose that $\lambda_{1} \in \Lambda^{*}, \lambda_{1}<\lambda_{2}$ and $\lambda_{2} \in \Lambda$. To derive the estimates in (4.4), we consider the difference of the solutions $\left|\phi_{\lambda_{1}}-\phi_{\lambda_{2}}\right|$ and $\left|\psi_{\lambda_{1}}-\psi_{\lambda_{2}}\right|$.

From the $\phi$-equations, we have

$$
\begin{aligned}
\Delta\left(\phi_{\lambda_{1}}^{i}-\phi_{\lambda_{2}}^{i}\right)= & -\tau \Gamma_{j k}^{i}\left(\phi_{\lambda_{2}}\right) \phi_{\lambda_{2}, \alpha}^{j} \phi_{\lambda_{2}, \beta}^{k} g^{\alpha \beta}+\tau \Gamma_{j k}^{i}\left(\phi_{\lambda_{1}}\right) \phi_{\lambda_{1}, \alpha}^{j} \phi_{\lambda_{1}, \beta}^{k} g^{\alpha \beta} \\
& -\frac{\tau}{2} R_{j k l}^{i}\left(\phi_{\lambda_{2}}\right)\left\langle\psi_{\lambda_{2}}^{k}, \nabla \phi_{\lambda_{2}}^{j} \cdot \psi_{\lambda_{2}}^{l}\right\rangle+\frac{\tau}{2} R_{j k l}^{i}\left(\phi_{\lambda_{2}}\right)\left\langle\psi_{\lambda_{1}}^{k}, \nabla \phi_{\lambda_{1}}^{j} \cdot \psi_{\lambda_{1}}^{l}\right\rangle .
\end{aligned}
$$

The boundary conditions of $\phi_{1}, \phi_{2}$ imply

$$
\left(\phi_{\lambda_{2}}-\phi_{\lambda_{1}}\right)-\tau\left(\lambda_{2}-\lambda_{1}\right) \phi_{0} \in\left(H_{0}^{2, p}(M)\right)^{n^{\prime}} .
$$

By the standard elliptic estimates with boundary conditions, we have

$$
\begin{aligned}
\left\|\phi_{\lambda_{2}}-\phi_{\lambda_{1}}\right\|_{2, p} & \leq C\left(\left\|\left|d \phi_{\lambda_{2}}\right|^{2}\right\|_{L^{p}}+\left\|\left|d \phi_{\lambda_{1}}\right|^{2}\right\|_{L^{p}}+\left\|\tau\left(\lambda_{2}-\lambda_{1}\right) \phi_{0}\right\|_{2, p}+\sqrt{R}+C_{0}^{2}\right) \\
& \leq C\left(\left\|\left|d \phi_{\lambda_{2}}-d \phi_{\lambda_{1}}\right|^{2}\right\|_{L^{p}}+\left\|\left|d \phi_{\lambda_{1}}\right|^{2}\right\|_{L^{p}}+\left\|\phi_{0}\right\|_{2, p}+\sqrt{R}+C_{0}^{2}\right) .
\end{aligned}
$$

Using the extended Sobolev inequality (see Theorem (10.1) in [11], p.27)

$$
\left\|\left|d \phi_{\lambda_{a}}\right|^{2}\right\|_{L^{p}} \leq C\left\|\phi_{\lambda_{a}}\right\|_{2, p}\left\|\phi_{\lambda_{a}}\right\|_{L^{\infty}}, \quad a=1,2
$$

in (4.7) we have

$$
\begin{aligned}
\left\|\phi_{\lambda_{2}}-\phi_{\lambda_{1}}\right\|_{2, p} \leq & C\left(\left\|\phi_{\lambda_{2}}-\phi_{\lambda_{1}}\right\|_{2, p}\left\|\phi_{\lambda_{2}}-\phi_{\lambda_{1}}\right\|_{L^{\infty}}\right. \\
& \left.+\left\|\phi_{\lambda_{1}}\right\|_{2, p}\left\|\phi_{\lambda_{1}}\right\|_{L^{\infty}}+\left\|\phi_{0}\right\|_{2, p}+\sqrt{R}+C_{0}^{2}\right) .
\end{aligned}
$$

On the other hand, from (1.14) in Theorem 1.1,

$$
\begin{aligned}
\left\|\phi_{\lambda_{2}}-\phi_{\lambda_{1}}\right\|_{L^{\infty}(M)} & \leq C\left(\left\|\phi_{\lambda_{2}}-\phi_{\lambda_{1}}\right\|_{H^{2-\frac{1}{p}, p}(\partial M)}+\left\|\mathbf{B} \psi_{1}-\mathbf{B} \psi_{2}\right\|_{H^{1-\frac{1}{p}, p}(\partial M)}\right) \\
& \leq C\left(\left\|\phi_{0}\right\|_{H^{2-\frac{1}{p}, p}(\partial M)}+\left\|\mathbf{B} \psi_{0}\right\|_{H^{1-\frac{1}{p}, p}(\partial M)}\right)\left|\lambda_{2}-\lambda_{1}\right| .
\end{aligned}
$$

From (4.9) and (4.10), we can find a positive constant $\delta=\delta\left(n, n^{\prime}, p, \phi_{0}, \psi_{0}, y_{0}, R\right.$, $C_{0}, M, N$ ) which is independent of $\lambda_{1}$ and $\tau$ such that if

$$
\left|\lambda_{1}-\lambda_{2}\right| \leq \delta
$$

then

$$
\left\|\phi_{\lambda_{2}}-\phi_{\lambda_{1}}\right\|_{2, p} \leq C\left(\left\|\phi_{\lambda_{1}}\right\|_{2, p}\left\|\phi_{\lambda_{1}}\right\|_{L^{\infty}}+\left\|\phi_{0}\right\|_{2, p}+\sqrt{R}+C_{0}^{2}\right),
$$

and consequently, for any $\lambda_{1} \in \Lambda^{*}$ and $\lambda_{2} \in\left[0, \lambda_{1}+\delta\right] \cap \Lambda$, we have

$$
\left\|\phi_{\lambda_{2}}\right\|_{2, p} \leq C\left(n, n^{\prime}, p, \phi_{0}, \psi_{0}, y_{0}, R, C_{0}, M, N, \lambda_{1}\right) .
$$


Now we derive estimates for the spinor fields $\psi$. From the $\psi$-equations, we have

$$
\left\{\begin{array}{l}
\not \partial\left(\psi_{\lambda_{2}}^{i}-\psi_{\lambda_{2}}^{i}\right)=-\tau \Gamma_{j k}^{i}\left(\phi_{\lambda_{2}}\right) \nabla \phi_{\lambda_{2}}^{j} \cdot \psi_{\lambda_{2}}^{k}+\tau \Gamma_{j k}^{i}\left(\phi_{\lambda_{1}}\right) \nabla \phi_{\lambda_{1}}^{j} \cdot \psi_{\lambda_{1}}^{k}, \\
\mathbf{B}\left(\psi_{\lambda_{2}}-\psi_{\lambda_{2}}-\tau\left(\lambda_{2}-\lambda_{1}\right) \psi_{0}\right)=0, \quad i=1,2, \cdots, n^{\prime} .
\end{array}\right.
$$

By Lemma 2.3 and our assumptions in Theorem 1.2, we have

$$
\left\|\psi_{\lambda_{2}}^{i}-\psi_{\lambda_{1}}^{i}-\tau\left(\lambda_{2}-\lambda_{1}\right) \psi_{0}^{i}\right\|_{1, p} \leq C\left\|\partial\left(\psi_{\lambda_{2}}^{i}-\psi_{\lambda_{1}}^{i}-\tau\left(\lambda_{2}-\lambda_{1}\right) \psi_{0}^{i}\right)\right\|_{L^{p}},
$$

for $i=1,2, \cdots, n^{\prime}$. Hence,

$$
\begin{aligned}
\left\|\psi_{\lambda_{2}}^{i}-\psi_{\lambda_{1}}^{i}\right\|_{1, p} & \leq C\left(\left\|\partial\left(\psi_{\lambda_{2}}^{i}-\psi_{\lambda_{1}}^{i}\right)\right\|_{L^{p}}+\tau\left|\lambda_{2}-\lambda_{1}\right|\left\|\psi_{0}\right\|_{1, p}\right) \\
& \leq C\left(\left\|\left|d \phi_{\lambda_{2}}\left\|\psi_{\lambda_{2}}\left|\left\|_{L^{p}}+\right\|\right| d \phi_{\lambda_{1}}\right\| \psi_{\lambda_{1}}\right|\right\|_{L^{p}}+\left\|\psi_{0}\right\|_{1, p}\right) \\
& \leq C\left(\left\|\left|d \phi_{\lambda_{2}}\right|^{2}\right\|_{L^{p}}+\left\|\left|d \phi_{\lambda_{1}}\right|^{2}\right\|_{L^{p}}+C_{0}+\mid \psi_{0} \|_{1, p}\right) .
\end{aligned}
$$

¿From the previous estimates (4.12), we have

$$
\left\|\psi_{\lambda_{2}}\right\|_{1, p} \leq C\left(n, n^{\prime}, p, \phi_{0}, \psi_{0}, y_{0}, R, C_{0}, M, N, \lambda_{1}\right) .
$$

The estimates (4.12) and (4.15) imply that

$$
\left[0, \lambda_{1}+\delta\right] \cap\{\lambda \mid[0, \lambda] \subset \Lambda\} \subset \Lambda^{*} .
$$

Next, we will show that $\left[0, \lambda_{1}+\delta\right] \subset \Lambda^{*}$. To see this, we first note that by (4.12) and (4.15) we have

$$
\left\{\begin{array}{l}
\left\|\phi_{\bar{\lambda}}\right\|_{2, p}+\left\|\psi_{\tilde{\lambda}}\right\|_{1, p} \leq C\left(n, n^{\prime}, p, \phi_{0}, \psi_{0}, y_{0}, R, C_{0}, M, N, \lambda_{1}\right):=\hat{c}, \\
\widetilde{\lambda} \in\left[0, \lambda_{1}+\delta\right] \cap \Lambda .
\end{array}\right.
$$

Similar to (4.10), for any solution $(\phi, \psi)$ of $P_{R, C_{0}}^{\tau, \lambda}\left(\phi_{0}, \psi_{0}\right)$, there is a positive constant $T_{0}=T_{0}\left(n, n^{\prime}, p, y_{0}, R, C_{0}, M, N\right)$ such that

$$
\|\phi\|_{L^{\infty}(M)}+\|\psi\|_{L^{\infty}(M)} \leq T_{0}\left(\left\|\phi_{0}\right\|_{H^{2-\frac{1}{p}, p}(\partial M)}+\left\|\mathbf{B} \psi_{0}\right\|_{H^{1-\frac{1}{p}, p}(\partial M)}\right) .
$$

For $R$ and $C_{0}$ already chosen, we can find small constants $a_{0}$ and $b_{0}$ such that when $\left\|\phi_{0}\right\|_{H^{2, p}(M)}<a_{0}$ and $\left\|\psi_{0}\right\|_{H^{1, p}(M)}<b_{0}$, we have

$$
T_{0}\left(\left\|\phi_{0}\right\|_{H^{2-\frac{1}{p}, p}(\partial M)}+\left\|\psi_{0}\right\|_{H^{1-\frac{1}{p}, p}(\partial M)}\right)<\min \left\{\sqrt{R} / E_{2}, \sqrt{C_{0}} / E_{2}\right\} .
$$

Set $\gamma:=1-\frac{n}{p} \in(0,1)$. Define

$$
\begin{gathered}
K_{1}:=\left\{\phi \in\left(C^{1+\gamma}(M)\right)^{n^{\prime}} \mid\|\phi\|_{\left(C^{0}(M)\right)^{n^{\prime}}}<A_{1},\|\phi\|_{\left(C^{1+\gamma}(M)\right)^{n^{\prime}}}<B\right\}, \\
K_{2}:=\left\{\psi \in\left(C^{\gamma}(\Sigma M)\right)^{n^{\prime}} \mid\|\psi\|_{\left(C^{0}(\Sigma M)\right)^{n^{\prime}}}<A_{2},\|\psi\|_{\left(C^{\gamma}(\Sigma M)\right)^{n^{\prime}}}<B\right\},
\end{gathered}
$$

where

$$
\left\{\begin{array}{l}
A_{1}<\sqrt{R} / E_{2}, \quad A_{2}<\sqrt{C_{0}} / E_{2}, \quad B>\hat{c}, \\
T_{0}\left(\left\|\phi_{0}\right\|_{H^{2-\frac{1}{p}, p}(\partial M)}+\left\|\psi_{0}\right\|_{H^{1-\frac{1}{p}, p}(\partial M)}\right)<\min \left\{A_{1}, A_{2}\right\} .
\end{array}\right.
$$


Then $K:=K_{1} \times K_{2}$ is a convex neighborhood of $0 \in\left(C^{1+\gamma}(M)\right)^{n^{\prime}} \times\left(C^{\gamma}(\Sigma M)\right)^{n^{\prime}}$. For any $(\Phi, \Psi) \in \bar{K}$, let $T^{\tau}(\Phi, \Psi) \equiv\left(T^{\tau} \Phi, T^{\tau} \Psi\right)$ be the unique solution of the following boundary value problem:

$$
\begin{gathered}
\left\{\begin{array}{l}
\Delta\left(T^{\tau} \Phi\right)^{i}+\tau \Gamma_{j k}^{i}(\Phi) \Phi_{\alpha}^{j} \Phi_{\beta}^{k} g^{\alpha \beta}+\frac{\tau}{2} R_{j k l}^{i}(\Phi)\left\langle\Psi^{k}, \nabla \Phi^{j} \cdot \Psi^{l}\right\rangle=0, \\
\partial\left(T^{\tau} \Psi\right)^{i}+\tau \Gamma_{j k}^{i}(\Phi) \nabla \Phi^{j} \cdot \Psi^{k}=0, \quad i=1,2, \cdots, n^{\prime} .
\end{array}\right. \\
\left\{\begin{array}{l}
T^{\tau} \Phi-\tau \lambda \phi_{0} \in\left(H_{0}^{2, p}(M)\right)^{n^{\prime}}, \\
\mathbf{B}\left(T^{\tau} \Psi-\tau \lambda \psi_{0}\right)=0 .
\end{array}\right.
\end{gathered}
$$

By the standard elliptic estimates and the fact that $H^{2, p}$ (resp. $H^{1, p}$ ) is compactly embedded into $C^{1+\gamma}$ (resp. $C^{\gamma}$ ), we have a compact map

$$
\begin{gathered}
T^{\tau}: \bar{K} \rightarrow\left(C^{1+\gamma}(M)\right)^{n^{\prime}} \times\left(C^{\gamma}(\Sigma M)\right)^{n^{\prime}}, \\
(\Phi, \Psi) \mapsto\left(T^{\tau} \Phi, T^{\tau} \Psi\right) .
\end{gathered}
$$

Define a map

$$
T^{\tau *}: \bar{K} \rightarrow\left(C^{1+\gamma}(M)\right)^{n^{\prime}} \times\left(C^{\gamma}(\Sigma M)\right)^{n^{\prime}}
$$

as follows:

$$
T^{\tau *} x:= \begin{cases}T^{\tau} x, & \text { if } T^{\tau} x \in \bar{K}, \\ x_{0}, & \text { if } T^{\tau} x \bar{\in} \bar{K},\end{cases}
$$

where $T^{\tau} x \equiv \sigma x_{0}$ with $x_{0} \in \partial K, \sigma \in[0,+\infty)$. It is clear that $T^{\tau *} x: \bar{K} \rightarrow \bar{K}$. By the Leray-Schauder fixed point theorem (c.f. Corollary 11.2 in [13]), $T^{\tau *}$ has a fixed point $x \in \bar{K}$, namely, $T^{\tau *} x=x$.

If $T^{\tau} x \bar{\in} \bar{K}$, then we have

$$
T^{\tau} x_{0}=\sigma x_{0}, \quad \sigma>1, \quad x_{0} \in \partial K .
$$

Denote $x_{0}:=\left(\Phi_{0}, \Psi_{0}\right)$, then (4.24) implies

$$
\begin{gathered}
\left\{\begin{array}{l}
\Delta \Phi_{0}^{i}+\frac{\tau}{\sigma} \Gamma_{j k}^{i}\left(\Phi_{0}\right) \Phi_{0, \alpha}^{j} \Phi_{0, \beta}^{k} g^{\alpha \beta}+\frac{\tau}{2 \sigma} R_{j k l}^{i}\left(\Phi_{0}\right)\left\langle\Psi_{0}^{k}, \nabla \Phi_{0}^{j} \cdot \Psi_{0}^{l}\right\rangle=0, \\
\not \partial \Psi_{0}^{i}+\frac{\tau}{\sigma} \Gamma_{j k}^{i}\left(\Phi_{0}\right) \nabla \Phi_{0}^{j} \cdot \Psi_{0}^{k}=0, \quad i=1,2, \cdots, n^{\prime} .
\end{array}\right. \\
\left\{\begin{array}{l}
\Phi_{0}-\frac{\tau}{\sigma} \lambda \phi_{0} \in\left(H_{0}^{2, p}(M)\right)^{n^{\prime}} \\
\mathbf{B}\left(\Psi_{0}-\frac{\tau}{\sigma} \lambda \psi_{0}\right)=0 .
\end{array}\right.
\end{gathered}
$$

Because $x_{0} \in \partial K$, at least one of the following four equalities $\left\|\Phi_{0}\right\|_{C^{0}}=A_{1},\left\|\Psi_{0}\right\|_{C^{0}}=A_{2}$, $\left\|\Phi_{0}\right\|_{C^{1+\gamma}}=B,\left\|\Psi_{0}\right\|_{C^{\gamma}}=B$ holds. From the estimates (4.17) and the assumptions in (4.20), we conclude that neither of the last two equalities can hold true. For the first two equalities, if $\left\|\Phi_{0}\right\|_{C^{0}}=A_{1}$, then by (4.18), we have

$$
\left\|\Phi_{0}\right\|_{C^{0}} \leq T_{0}\left(\left\|\phi_{0}\right\|_{H^{2-\frac{1}{p}, p}(\partial M)}+\left\|\mathbf{B} \psi_{0}\right\|_{H^{1-\frac{1}{p}, p}(\partial M)}\right),
$$

from (4.20), $\left\|\Phi_{0}\right\|_{C^{0}}<A_{1}$, a contradiction. Similarly, $\left\|\Psi_{0}\right\|_{C^{0}}=A_{2}$ can not hold. Therefore, we must have $T^{\tau} x \in \bar{K}$. Consequently, $T^{\tau} x=x$. We have proved that for all $\lambda \in\left[0, \lambda_{1}+\delta\right]$ and $\tau \in[0,1]$, the boundary value problem $P_{R, C_{0}}^{\tau, \lambda}\left(\phi_{0}, \psi_{0}\right)$ admits a solution. In other words, $\left[0, \lambda_{1}+\delta\right] \subset \Lambda^{*}$. Since $\delta$ is independent of $\lambda_{1}$, through the above argument step by step, we conclude that $\Lambda^{*}=[0,1]$. This completes the proof of Theorem 1.2. 


\section{A Appendix}

Proof of lemma 3.1. The strategy will be to control $\phi$ in terms of estimates for a harmonic map $\phi_{0}$ with the same boundary values plus some results from the theory of quasilinear elliptic systems. Thus, we choose a harmonic map $\widetilde{\phi_{0}}: M \rightarrow B_{R}\left(y_{0}\right)$ with $\left.\widetilde{\phi_{0}}\right|_{\partial M}=\left.\phi\right|_{\partial M}$. Then by the theory of harmonic maps, we have

$$
\left|\nabla \widetilde{\phi}_{0}\right|, \quad\left|\nabla^{2} \widetilde{\phi}_{0}\right| \leq C .
$$

Set $\widetilde{\phi}:=\phi-\widetilde{\phi}_{0}$, then $\widetilde{\phi}$ satisfies

$$
g^{\alpha \beta} \widetilde{\phi}_{\alpha \beta}^{i}+b_{\gamma}(x) \widetilde{\phi}_{\gamma}^{i}+b^{i}(x, \widetilde{\phi}, d \widetilde{\phi})=0
$$

with

$$
\begin{gathered}
b_{\gamma}(x):=-\Gamma_{\alpha \beta}^{\gamma}(x) g^{\alpha \beta}(x), \quad \gamma=1,2, \cdots, n, \\
b^{i}(x, \widetilde{\phi}, d \widetilde{\phi}):=\Gamma_{j k}^{i}(\phi) \widetilde{\phi}_{\alpha}^{j} \widetilde{\phi}_{\beta}^{k} g^{\alpha \beta}-\frac{1}{2} R_{j k l}^{i}(\phi)\left\langle\nabla \widetilde{\phi}^{j} \cdot \psi^{k}, \psi^{l}\right\rangle \\
+\Gamma_{j k}^{i}(\phi) \widetilde{\phi}_{\alpha}^{j} \widetilde{\phi}_{0 \beta}^{k} g^{\alpha \beta}+\Gamma_{j k}^{i}(\phi) \widetilde{\phi}_{0 \alpha}^{j} \widetilde{\phi}_{\beta}^{k} g^{\alpha \beta}-\frac{1}{2} R_{j k l}^{i}(\phi)\left\langle\nabla \widetilde{\phi}_{0}^{j} \cdot \psi^{k}, \psi^{l}\right\rangle,
\end{gathered}
$$

$i=1,2, \cdots, n^{\prime}$. Denote $\mathbf{b}(x, \widetilde{\phi}, d \widetilde{\phi}):=\left(b^{1}(x, \widetilde{\phi}, d \widetilde{\phi}), \cdots, b^{n^{\prime}}(x, \widetilde{\phi}, d \widetilde{\phi})\right)$, it's easy to see that the elliptic system (A.2) satisfies the following structure conditions:

$$
\begin{aligned}
& \text { (1) } v \sum_{\alpha} \xi_{\alpha}^{2} \leq g^{\alpha \beta} \xi_{\alpha} \xi_{\beta} \leq \mu \sum_{\alpha} \xi_{\alpha}^{2}, \\
& \text { (2) }\left|b_{\gamma}\right| \leq \mu, \\
& \text { (3) }|\mathbf{b}(x, \widetilde{\phi}, d \widetilde{\phi})| \leq \varepsilon\left(R, C_{0}\right)\left(1+|d \widetilde{\phi}|^{2}\right), \\
& \text { (4) }\left|\frac{\partial g^{\alpha \beta}}{\partial x^{\gamma}}\right| \leq \mu,
\end{aligned}
$$

where $v, \mu$ and $\varepsilon\left(R, C_{0}\right)$ are positive constants, and $\varepsilon\left(R, C_{0}\right)$ is small when $R$ and $C_{0}$ are small. By Theorem 4.1 in [27] (p.417) and then by the standard elliptic estimates with boundary, we have (3.15).

Proof of (3.20) and (3.21). These will be derived from (3.9) by some easy inequalities. We recall

$$
\begin{aligned}
J= & -\left\langle\left(\Gamma_{j k}^{i}\left(\phi_{2}\right)-\Gamma_{j k}^{i}\left(\phi_{1}\right)\right) \Delta \phi_{2}^{j} \psi_{2}^{k}, \psi_{1}^{i}-\psi_{2}^{i}\right\rangle-\left\langle\Gamma_{j k}^{i}\left(\phi_{1}\right)\left(\Delta \phi_{2}^{j}-\Delta \phi_{1}^{j}\right) \psi_{2}^{k}, \psi_{1}^{i}-\psi_{2}^{i}\right\rangle \\
& -\left\langle\Gamma_{j k}^{i}\left(\phi_{1}\right) \Delta \phi_{1}^{j}\left(\psi_{2}^{k}-\psi_{1}^{k}\right), \psi_{1}^{i}-\psi_{2}^{i}\right\rangle \\
:= & J_{1}+J_{2}+J_{3} .
\end{aligned}
$$

Recalling that

$$
\begin{aligned}
\Delta \phi_{a}^{i} & =-\Gamma_{j k}^{i}\left(\phi_{a}\right) \phi_{a \alpha}^{j} \phi_{a \beta}^{k} g^{\alpha \beta}+\mathcal{R}^{i}\left(\phi_{a}, \psi_{a}\right), \\
& =-\Gamma_{j k}^{i}\left(\phi_{a}\right) \phi_{a \alpha}^{j} \phi_{a \beta}^{k} g^{\alpha \beta}+\frac{1}{2} R_{j k l}^{i}\left(\phi_{a}\right)\left\langle\nabla \phi_{a}^{j} \cdot \psi_{a}^{k}, \psi_{a}^{l}\right\rangle,
\end{aligned}
$$


$a=1,2, \quad i=1,2, \cdots, n^{\prime}$, we have

$$
\begin{aligned}
\left|J_{1}\right| & \leq C_{S} \rho\left(\sum_{a}\left|d \phi_{a}\right|^{2}+\sum_{a}\left|\psi_{a}\right|^{4}\right)\left|\psi_{1}-\psi_{2}\right| \\
& \leq C_{S} \widetilde{\xi}\left(\sum_{a}\left|d \phi_{a}\right|^{2}+\sum_{a}\left|\psi_{a}\right|^{4}\right) .
\end{aligned}
$$

Since

$$
\left|J_{2}\right| \leq C_{S} \sum_{i}\left|\Delta \phi_{1}^{i}-\Delta \phi_{2}^{i}\right|\left|\psi_{2}\right|\left|\psi_{1}-\psi_{2}\right|,
$$

and using (3.9) we have, for $i=1,2, \cdots, n^{\prime}$,

$$
\begin{aligned}
\left|\Delta \phi_{1}^{i}-\Delta \phi_{2}^{i}\right| \leq & \mid\left(\Gamma_{j k}^{i}\left(\phi_{2}\right)-\Gamma_{j k}^{i}\left(\phi_{1}\right)\right) \phi_{2 \alpha}^{j} \phi_{2 \beta}^{k} g^{\alpha \beta}+\Gamma_{j k}^{i}\left(\phi_{1}\right)\left(\phi_{2 \alpha}^{j}-\phi_{1 \alpha}^{j}\right) \phi_{2 \beta}^{k} g^{\alpha \beta} \\
& +\Gamma_{j k}^{i}\left(\phi_{1}\right) \phi_{1 \alpha}^{j}\left(\phi_{2 \beta}^{k}-\phi_{1 \beta}^{k}\right) g^{\alpha \beta}|+| \mathcal{R}_{1}-\mathcal{R}_{2} \mid \\
\leq & C \rho\left|d \phi_{2}\right|^{2}+C_{S}\left|d \phi_{1}-d \phi_{2}\right|\left|d \phi_{2}\right|+C \rho\left|d \phi_{1}\right|\left|\psi_{1}\right|^{2} \\
& +C\left|d \phi_{1}-d \phi_{2}\right|\left|\psi_{1}\right|^{2}+C\left|d \phi_{2}\right|\left(\left|\psi_{1}\right|+\left|\psi_{2}\right|\right)\left|\psi_{1}-\psi_{2}\right|,
\end{aligned}
$$

hence,

$$
\begin{aligned}
\left|J_{2}\right| \leq & C_{S} \rho\left|d \phi_{2}\right|^{2}\left|\psi_{2}\right|\left|\psi_{1}-\psi_{2}\right|+C_{S}\left|\psi_{2}\right|\left|d \phi_{2}\right|\left|d \phi_{1}-d \phi_{2}\right|\left|\psi_{1}-\psi_{2}\right| \\
& +C_{S} \rho\left|d \phi_{1}\right|\left|\psi_{1}\right|^{2}\left|\psi_{2}\right|\left|\psi_{1}-\psi_{2}\right|+C_{S}\left|\psi_{1}\right|^{2}\left|\psi_{2}\right|\left|d \phi_{1}-d \phi_{2}\right|\left|\psi_{1}-\psi_{2}\right| \\
& +C_{S}\left|d \phi_{2}\right|\left|\psi_{2}\right|\left(\left|\psi_{1}\right|+\left|\psi_{2}\right|\right)\left|\psi_{1}-\psi_{2}\right|^{2} \\
\leq & C_{S} \widetilde{\xi} \sum_{a}\left|d \phi_{a}\right|^{2}+C_{S} \delta^{2}\left(d \phi_{1}, d \phi_{2}\right)+C_{S}\left|\psi_{1}-\psi_{2}\right|^{2} .
\end{aligned}
$$

Furthermore,

$$
\begin{aligned}
\left|J_{3}\right| & \leq C_{S}\left|\Delta \phi_{1}\right|\left|\psi_{1}-\psi_{2}\right|^{2} \\
& \leq C_{S}\left(\left|d \phi_{1}\right|^{2}+\left|d \phi_{1}\right|\left|\psi_{1}\right|^{2}\right)\left|\psi_{1}-\psi_{2}\right|^{2} \\
& \leq C_{S} \widetilde{\xi} \sum_{a}\left(\left|d \phi_{a}\right|^{2}+\left|\psi_{a}\right|^{4}\right)
\end{aligned}
$$

hence, we obtain

$$
|J| \leq C_{S} \widetilde{\xi} \sum_{a}\left(\left|d \phi_{a}\right|^{2}+\left|\psi_{a}\right|^{4}\right)+C_{S} \delta^{2}\left(d \phi_{1}, d \phi_{2}\right)+C_{S}\left|\psi_{1}-\psi_{2}\right|^{2} .
$$

Finally,

$$
\begin{aligned}
K= & \left\langle\left(\Gamma_{j k}^{i}\left(\phi_{2}\right)-\Gamma_{j k}^{i}\left(\phi_{1}\right)\right) e_{\alpha} \cdot \nabla \phi_{2}^{j} \cdot \psi_{2 \alpha}^{k}, \psi_{1}^{i}-\psi_{2}^{i}\right\rangle \\
& +\left\langle\Gamma_{j k}^{i}\left(\phi_{1}\right) e_{\alpha} \cdot\left(\nabla \phi_{2}^{j}-\nabla \phi_{1}^{j}\right) \cdot \psi_{2 \alpha}^{k}, \psi_{1}^{i}-\psi_{2}^{i}\right\rangle \\
& +\left\langle\Gamma_{j k}^{i}\left(\phi_{1}\right) e_{\alpha} \cdot \nabla \phi_{1}^{j} \cdot\left(\psi_{2 \alpha}^{k}-\psi_{1 \alpha}^{k}\right), \psi_{1}^{i}-\psi_{2}^{i}\right\rangle,
\end{aligned}
$$

we have

$$
\begin{aligned}
|K| \leq & C \rho\left|d \phi_{2}\right|\left|\nabla \psi_{2}\right|\left|\psi_{1}-\psi_{2}\right|+C_{S}\left|d \phi_{1}-d \phi_{2}\right|\left|\nabla \psi_{2}\right|\left|\psi_{1}-\psi_{2}\right| \\
& +C_{S}\left|d \phi_{1}\right|\left|\nabla\left(\psi_{1}-\psi_{2}\right)\right|\left|\psi_{1}-\psi_{2}\right|
\end{aligned}
$$




$$
\begin{aligned}
\leq & C_{S} \delta^{2}\left(d \phi_{1}, d \phi_{2}\right)+\frac{C}{\varepsilon} \widetilde{\xi} \sum_{a}\left|d \phi_{a}\right|^{2}+C_{S}\left|\nabla\left(\psi_{1}-\psi_{2}\right)\right|^{2} \\
& +\left(C \varepsilon+C_{S}\right)\left|\psi_{1}-\psi_{2}\right|^{2}
\end{aligned}
$$

for any small constant $\varepsilon>0$.

Acknowledgments The research leading to these results has received funding from the European Research Council under the European Union's Seventh Framework Programme (FP7/2007-2013) / ERC grant agreement no. 267087. The research of QC is also partially supported by NSFC and RFDP of China. The authors thank the Max Planck Institute for Mathematics in the Sciences for good working conditions when this work was carried out.

Open Access This article is distributed under the terms of the Creative Commons Attribution License which permits any use, distribution, and reproduction in any medium, provided the original author(s) and the source are credited.

\section{References}

1. Atiyah, M.F., Patodi, V.K., Singer, I.M.: Spectral asymmetry and Riemannian geometry, I, II and III. Math. Proc. Camb. Phil. Soc. 77, 43-69 (1975)

2. Atiyah, M.F., Patodi, V.K., Singer, I.M.: Spectral asymmetry and Riemannian geometry, I, II and III. Math. Proc. Camb. Phil. Soc. 78, 405-432 (1975)

3. Atiyah, M.F., Patodi, V.K., Singer, I.M.: Spectral asymmetry and Riemannian geometry, I, II and III. Math. Proc. Camb. Phil. Soc. 79, 71-99 (1975)

4. Bär, C.: Extrinsic bounds of the Dirac operator. Ann. Glob. Anal. Geom. 16, 573-596 (1998)

5. Booß-Bavnbek, B., Wojciechowski, K.P.: Elliptic boundary problems for the Dirac operator. Birkhaüser, Basel (1993)

6. Baum, H., Friedrich, T., Grünewald, R., Kath, I.: Twistor and Killing spinors on Riemannian manifolds, Teubner-Texte zur Mathematik, Band 124, B.G.Teubner Verlagsgesellshaft, Stuttgart (1991)

7. Brüning, J., Lesch, M.: Spectral theory of boundary value problems for Dirac type operators. Contemp. Math. 242, 203-215 (1999)

8. Chen, Q., Jost, J., Li, J.Y., Wang, G.: Dirac-harmonic maps. Math. Z 254, 409-432 (2006)

9. Chen, Q., Jost, J., Li, J.Y., Wang, G.F.: Regularity theorem and energy identities for Dirac-harmonic maps. Math. Z 251, 61-84 (2005)

10. Chen, Q., Jost, J., Wang, G., Zhu. M.M.: Boundary value problems for Dirac-harmonic maps. J. Eur. Math. Soc (2012)

11. Friedman, A.: Partial differential equations. Holt, Rinihart and Winston, Inc., New York (1969)

12. Farinelli, S., Schwarz, G.: On the spectrum of the Dirac operator under boundary conditions. J. Geom. Phys. 28, 67-84 (1998)

13. Gilbarg, D., Trudinger, N.S.: Elliptic partial differential equations of second order, 2nd Edition. Springer, Berlin (1983)

14. Hijazi, O., Montiel, S., Roldán, A.: Eigenvalue boundary problems for the Dirac operator.Comm. Math. Phys. 231(3), 375-390 (2002)

15. Hildebrandt, S., Jost, J., Widman, K.O.: Harmonic mappings and minimal submanifolds. Inv. Math. 62, 269-298 (1980)

16. Hildebrandt, S., Kaul, H., Widman, K.O.: An existence theorem for harmonic mappings of Riemannian manifolds. Acta Math. 138, 1-16 (1977)

17. Johnson, K.: The M.I.T. bag model. Acta Phys. Pol B 6, 865-892 (1975)

18. Jäger, W., Kaul, H.: Uniqueness of harmonic mappings and of solutions of elliptic equations on Riemannian manifolds. Math. Ann. 240, 231-250 (1979)

19. Jäger, W., Kaul, H.: Uniqueness and stability of harmonic maps and their Jacobi fields. Manuscr. Math. 28, 269-291 (1979)

20. Jost, J.: Harmonic mappings between Riemannian manifolds. ANU-Press, Canberra (1984)

21. Jost, J.: Riemannian geometry and geometric analysis, Fifth edition. Universitext. Springer, Berlin (2008)

22. Jost, J.: Geometry and physics, Springer, Berlin (2009)

23. Jost, J.: Two-dimensional geometric variational problems, Wiley, Chichester (1991) 
24. Jost, J.: Existence proofs for harmonic mappings with the help of a maximum principle. Math. Z 184, 489496 (1983)

25. Jost, J., Mo, X., Zhu, M.M.: Some explicit constructions of Dirac-harmonic maps. J. Geom. Phys. 59, 1512-1527 (2009)

26. Karcher, H.S.: Riemannian center of mass and mollifier smoothing. Commun. Pure Appl. Math. 30, 509541 (1977)

27. Ladyzhenskaya, O.A., Ural'tseva, N.N.: Linear and Quasilinear Elliptic Equations. Academic, New York (1968)

28. Schwarz, G.: Hodge Decompositiona Method for Solving Boundary Value Problems, Lecture Notes in Mathematics 1607, Springer, Berlin (1995)

29. Schoen, R., Yau, S.T.: Lectures on harmonic maps, Conference Proceedings and Lecture Notes in Geometry and Topology, II. International Press, Cambridge (1997)

30. Sacks, J., Uhlenbeck, K.: The existence of minimal immersions of 2-spheres. Ann. Math. 113(1), 1$24(1981)$

31. Wahl W.v.: Existenzsätze fr nichtlineare elliptische Systeme, Nachr. Akad. Wiss. Göttingen Math. Phys. Kl. II 53-62, (1978)

32. Wang, C.Y., Xu, D.L.: Regularity of Dirac-harmonic maps. Int. Math. Res. Not. 20, 3759-3792 (2009)

33. Yang, L.: A structure theorem of Dirac-harmonic maps between spheres. Calc. Var. Partial Differ. Equ. 35, 409-420 (2009)

34. Zhao, L.: Energy identities for Dirac-harmonic maps. Calc. Var. Partial Differ. Equ. 28, 121-138 (2007)

35. Zhu, M.M.: Regularity for weakly Dirac-harmonic maps to hypersurfaces. Ann. Glob. Anal. Geom. 35, 405-412 (2009) 